\title{
From Rational Gödel Logic to Ultrametric Logic
}

\author{
S. M. A. Khatami ${ }^{* 1}$, M. Pourmahdian ${ }^{\dagger 1}$ and N. R. Tavana ${ }^{\ddagger 2}$ \\ ${ }^{1}$ Department of Mathematics and Computer Science, Amirkabir \\ University of Technology, Tehran, Iran \\ ${ }^{2}$ School of Mathematics, Institute for Research in Fundamental \\ Sciences (IPM), Tehran, Iran
}

\begin{abstract}
This paper is devoted to systematic studies of some extensions of firstorder Gödel logic. The first extension is the first-order rational Gödel logic which is an extension of first-order Gödel logic, enriched by countably many nullary logical connectives. By introducing some suitable semantics and proof theory, it is shown that the first-order rational Gödel logic has the completeness property, that is any (strongly) consistent theory is satisfiable. Furthermore, two notions of entailment and strong entailment are defined and their relations with the corresponding notion of proof is studied. In particular, an approximate entailment-compactness is shown. Next, by adding a binary predicate symbol $d$ to the first-order rational Gödel logic, the ultrametric logic is introduced. This serves as a suitable framework for analyzing structures which carry an ultrametric function $d$ together with some functions and predicates which are uniformly continuous with respect to the ultrametric $d$. Some model theory is developed and to justify the relevance of this model theory, the Robinson joint consistency theorem is proven.
\end{abstract}

Keywords: First-order Gödel logic, Continuous metric logic, Ultrametric logic, Robinson consistency theorem

2010MSC: 03B50, 03C90

\section{Introduction}

The goal of this paper is twofold. On one hand, we are aiming to study some logical aspects of extensions of first-order Gödel logic and on the other hand, to introduce an alternative framework for (continuous) metric logic. The fuzzy logic is a branch of mathematical logic dealing with systematic studies of logical aspects of different types of many-valued logics, whose main emphasis rely mostly on introducing a relevant semantics for a given many-valued logic, exploring a (complete) set of axiomatic system and ultimately proving some kind of completeness results [10, 1]. The Łukasiewicz and Gödel logics [14, 6, 2, 8, 11]

\footnotetext{
*E-mail: amin_khatami@aut.ac.ir

${ }^{\dagger}$ Corresponding author E-mail: pourmahd@ipm.ir

‡E-mail: nazanin.r.tavana@ipm.ir
} 
are two important examples of fuzzy logics. The BL-algebras serve as the most general form of the set of truth values for different types of fuzzy logic [10]. However, in many important cases such as Łukasiewicz logic, it suffices to consider the standard truth valued set $[0,1]$. The semantics based on this set is called the standard semantics. If one considers the compact Euclidian topology on $[0,1]$, then one of the distinguished characters of the Eukasiewicz logic as well as its extension Pavelka logic is that all the logical connectives including implication are continuous functions. This simple but fundamental fact implies some of the important results including the Pavelka style completeness theorem for standard semantics of these logics [13, 10].

The starting step of this paper is to study the first-order rational Gödel logic. The relevance of this extension to Gödel logic is the same as the rational Pavelka logic to Lukasiewicz logic. To accomplish our goal, the first-order Gödel logic is enriched by countably many nullary connectives which are naturally interpreted by the rational numbers in the truth value set $[0,1]$ [9]. There are some major differences between the rational Gödel logic and Pavelka logic. The first major difference is that the standard semantics should be replaced by some "nonstandard" semantics. For this, one should first consider the set $\mathbb{I}=[0,1]^{2} \backslash$ $\{(0, r): r>0\}$ which is the set of truth values in rational Gödel logic. So, in this logic, the predicates are interpreted in the set $\mathbb{I}$. Secondly, the lack of the continuity of logical connectives with respect to the order topology on the set of truth values, which causes the loss of Pavelka style completeness of this logic.

The second step of this paper is to choose an alternative setting to extend the ideas from (continuous) metric logic to the first-order rational Gödel logic. A common trend in the current development of model theoretic investigations is to implement the logical ideas with an eye towards interesting mathematical structures. (Continuous) Metric logic [5, 3, 4, 7] aims to extend this new trend of model theory to a class of mathematical structures, which naturally arose in analysis. This research pass has already shed a new light on current studies in model theory. The logical formalism in which this model theory is based on is an important instant of many-valued logic, namely Łukasiewicz logic [12, 14, 6, 2, 7, 13. Loosely speaking, a metric structure in this logic consists of a (complete) metric space $(M, d: X \times X \rightarrow[0,1])$ together with some additional relations and functions which are uniformly continuous with respect to the metric function $d$. In this sense, the (continuous) metric logic is the model theory of (complete) metric structures.

The starting point in (continuous) metric logic relies heavily on this fact as how to modify the Eukasiewicz logic with the standard semantics so that the "fuzzy" interpretation of the equality relation coincides with the notion of metric [5].

An important subclass of metric spaces consists of ultrametric spaces. Recall that a metric space $(M, d)$ is an ultrametric if it satisfies a stronger form of the triangle inequality in which for any $a, b, c \in M$,

$$
d(a, b) \leq \max (d(a, c), d(c, b)) .
$$

So, in particular, an ultrametric structure can be defined in a similar fashion as above. In this sense, these structures can also be studied within the (continuous) metric logic.

If one modifies the Gödel logic in the same way as Eukasiewicz logic, the interpretation of the equality predicate coincides with the notion of ultramet- 
ric. That is why one may believe this is a natural approach towards studying ultrametric structures. To emphasize on this natural approach, this new logic is called the ultrametric logic.

This paper is organized as follows. The next section is devoted to study the first-order rational Gödel logic. First, by defining a suitable semantic and giving an axiomatization system, it is shown that the first-order rational Gödel logic satisfies that completeness theorem (Theorem 2.20). Then, two notions of entailment and strong entailment are discussed and their connections to the corresponding notion of proof are explored. In particular, it is proven that the first-order rational Gödel logic satisfies the approximate entailment compactness (Theorem 2.24).

The third and fourth sections include the key notion of ultrametric logic and some model theoretic machineries developed for proving the Robinson joint consistency theorem for this logic (Theorem 4.15).

The paper is concluded by given some guidelines as how to proceed this research further in order to explore the relevance of this logic for understanding the interesting mathematic structures.

\section{Rational Gödel Logic}

In this section the first-order rational Gödel logic is given. The syntactical issues of this logic is the same as the usual first-order Gödel logic. However, the semantical aspects which is given here are somewhat different from the usual approach if fuzzy logic. Opposite to the usual conventions in fuzzy logic, the nullary connective $\overline{0}$ is assumed as the absolute truth, while $\overline{1}$ as the absolute falsity. Moreover a formula is satisfied in a model $\mathcal{M}$ if its interpretation in $\mathcal{M}$ is zero. This approach is taken from the (continuous) metric logic and will be justified when the ultrametric logic is introduced. Furthermore, it would be very easy to see that all of the results in this section can be translated to usual semantical approach in (fuzzy) first-order Gödel logic.

Throughout this paper, suppose $\mathcal{L}$ is a first-order language with countably many predicate, function and constant symbols. As usual, we also assume a countable set of variables together with the set of boolean connectives $\{\vee, \wedge, \rightarrow, \neg, \overline{0}, \overline{1}\}$ and the set of quantifiers $\{\forall, \exists\}$. The rational Gödel logic $R G L^{*}$ is obtained by extending the set of nullary connectives $\{\overline{0}, \overline{1}\}$ with the set $\mathcal{A}=\left\{\bar{r}: r \in[0,1]_{\mathbb{Q}}=\mathbb{Q} \cap[0,1]\right\}$ of nullary connectives. The corresponding notions of $\mathcal{L}$-terms, (atomic) $\mathcal{L}$-formulas and subformulas are defined as usual. In particular, the notion of free variables, bound variables and sentences, i.e., formulas without free variables are considered as classical first-order logic. The set of $\mathcal{L}$-formulas and $\mathcal{L}$-sentences are denoted by $\operatorname{Form}(\mathcal{L})$ and $\operatorname{Sent}(\mathcal{L})$, respectively. For an $\mathcal{L}$-formula $\varphi, \operatorname{Sub}(\varphi)$ is the set of subformulas of $\varphi$. An $\mathcal{L}$-theory is an arbitrary set of $\mathcal{L}$-sentences.

Below, the semantical aspects of $R G L^{*}$ are introduced. Unlike the standard semantics, the set $\mathbb{I}=[0,1]^{2} \backslash\{(0, r): r>0\}$ with the lexicographical ordering is taken as the set of truth values, since as otherwise the compactness theorem fails if the standard set of truth values $[0,1]$ is assumed. For simplicity, we use the notation $\hat{r}$ instead of $(r, r) \in \mathbb{I}$.

Definition 2.1. An $\mathcal{L}$-structure $\mathcal{M}$ is a nonempty set $M$ called the universe of $\mathcal{M}$ together with: 
a) for any n-ary predicate symbol $P$ of $\mathcal{L}$, a function $P^{\mathcal{M}}: M^{n} \rightarrow \mathbb{I}$,

b) for any n-ary function symbol $f$ of $\mathcal{L}$, a function $f^{\mathcal{M}}: M^{n} \rightarrow M$,

c) for any constant symbol $c$ of $\mathcal{L}$, an element $c^{\mathcal{M}}$ in the universe of $\mathcal{M}$.

For each $\alpha \in \mathcal{L}, \alpha^{\mathcal{M}}$ is called the interpretation of $\alpha$ in $\mathcal{M}$.

An $\mathcal{M}$-assignment of variables is a function $\sigma$ from the set of variables into the set $M$. The interpretation of $\mathcal{L}$-terms is defined as follows.

Definition 2.2. Let $\mathcal{M}$ and $\sigma$ be as above and let $\bar{x}=\left(x_{1} \ldots x_{n}\right)$. Then, for every term $t(\bar{x})$,

1. if $t(\bar{x})=x_{i}$ for $1 \leq i \leq n$, then $t^{\mathcal{M}, \sigma}(\bar{x})=\sigma\left(x_{i}\right)$,

2. if $t(\bar{x})=c$ then $t^{\mathcal{M}, \sigma}(\bar{x})=c^{\mathcal{M}}$,

3. if $t(\bar{x})=f\left(t_{1}(\bar{x}), \ldots, t_{n}(\bar{x})\right)$ then $t^{\mathcal{M}, \sigma}(\bar{x})=f^{\mathcal{M}}\left(t_{1}^{\mathcal{M}, \sigma}(\bar{x}), \ldots, t_{n}^{\mathcal{M}, \sigma}(\bar{x})\right)$.

The interpretation of $\mathcal{L}$-formulas inside an $\mathcal{L}$-structure $\mathcal{M}$ is defined as follows.

Definition 2.3. Let $\mathcal{M}$ be an $\mathcal{L}$-structure and $\sigma$ be an $\mathcal{M}$-assignment.

1. for every $\bar{r} \in \mathcal{A}, \bar{r}^{\mathcal{M}, \sigma}=\hat{r}$. Particularly, $\overline{1}^{\mathcal{M}, \sigma}=\hat{1}$ and $\overline{0}^{\mathcal{M}, \sigma}=\hat{0}$.

2. $P^{\mathcal{M}, \sigma}\left(t_{1}(\bar{x}), \ldots, t_{n}(\bar{x})\right)=P^{\mathcal{M}}\left(t_{1}^{\mathcal{M}, \sigma}(\bar{x}), \ldots, t_{n}^{\mathcal{M}, \sigma}(\bar{x})\right)$.

3. $(\varphi \wedge \psi)^{\mathcal{M}, \sigma}(\bar{x})=\max \left\{\varphi^{\mathcal{M}, \sigma}(\bar{x}), \psi^{\mathcal{M}, \sigma}(\bar{x})\right\}$.

4. $(\varphi \rightarrow \psi)^{\mathcal{M}, \sigma}(\bar{x})=\left\{\begin{array}{cc}0 & \varphi^{\mathcal{M}, \sigma}(\bar{x}) \geq \psi^{\mathcal{M}, \sigma}(\bar{x}), \\ \psi^{\mathcal{M}, \sigma}(\bar{x}) & \varphi^{\mathcal{M}, \sigma}(\bar{x})<\psi^{\mathcal{M}, \sigma}(\bar{x}) .\end{array}\right.$

5. $(\forall x \varphi(x))^{\mathcal{M}, \sigma}=\sup \left\{\varphi^{\mathcal{M}, \sigma^{\prime}}(x): \sigma(x)=\sigma^{\prime}(x)\right\}$.

6. $(\exists x \varphi(x))^{\mathcal{M}, \sigma}=\inf \left\{\varphi^{\mathcal{M}, \sigma^{\prime}}(x): \sigma(x)=\sigma^{\prime}(x)\right\}$.

Note that 5 and 6 are well-defined, since $\mathbb{I}$ is a complete ordered set, i.e., any subset of $\mathbb{I}$ has the least upper bound and the greatest lower bound, respectively.

The following connectives $\neg, \vee, \leftrightarrow, \Rightarrow$ are given in terms of the above connectives.

$$
\begin{gathered}
\neg \varphi:=\varphi \rightarrow \overline{1} . \\
\varphi \vee \psi:=((\varphi \rightarrow \psi) \rightarrow \psi) \wedge((\psi \rightarrow \varphi) \rightarrow \varphi) . \\
\varphi \leftrightarrow \psi:=(\varphi \rightarrow \psi) \wedge(\psi \rightarrow \varphi) . \\
\varphi \Rightarrow \psi:=(\psi \rightarrow \varphi) \rightarrow \psi .
\end{gathered}
$$

Hence, their interpretations can be accordingly computed. In particular,

$$
\begin{aligned}
(\varphi \vee \psi)^{\mathcal{M}, \sigma}(\bar{x}) & =\min \left\{\varphi^{\mathcal{M}, \sigma}(\bar{x}), \psi^{\mathcal{M}, \sigma}(\bar{x})\right\}, \\
(\varphi \leftrightarrow \psi)^{\mathcal{M}, \sigma}(\bar{x}) & =d_{\max }\left(\varphi^{\mathcal{M}, \sigma}(\bar{x}), \psi^{\mathcal{M}, \sigma}(\bar{x})\right),
\end{aligned}
$$

where

$$
d_{\text {max }}(x, y)=\left\{\begin{array}{cc}
\max \{x, y\} & x \neq y \\
\hat{0} & x=y .
\end{array}\right.
$$


Observe that if $\psi^{\mathcal{M}, \sigma}(\bar{x})>\hat{0}$ then $(\varphi \Rightarrow \psi)^{\mathcal{M}, \sigma}(\bar{x})=\hat{0}$ iff $\varphi^{\mathcal{M}, \sigma}(\bar{x})>\psi^{\mathcal{M}, \sigma}(\bar{x})$. Therefore, $(\varphi(\bar{x}) \Rightarrow \bar{r})^{\mathcal{M}, \sigma}=\hat{0}$ for $r>0$ iff $\varphi^{\mathcal{M}, \sigma}(\bar{x})>\hat{r}$.

When $\varphi(\bar{x})$ is a quantifier free $\mathcal{L}$-formula, $\varphi^{\mathcal{M}, \sigma}(\bar{x})$ is dependent only on $\sigma(\bar{x})$. Thus, if $\sigma(\bar{x})=\bar{a} \in M^{n}$ we may write $\varphi^{\mathcal{M}}(\bar{a})$ instead of $\varphi^{\mathcal{M}, \sigma}(\bar{x})$. If $\varphi$ is an $\mathcal{L}$-sentence we write $\varphi^{\mathcal{M}}$ for $\varphi^{\mathcal{M}, \sigma}$.

Definition 2.4. Let $\mathcal{M}$ be an $\mathcal{L}$-structure. For an $\mathcal{L}$-formula $\varphi(\bar{x})$ and an $\mathcal{L}$-theory $T$,

1. $\varphi(\bar{x})$ is satisfied by $\bar{a} \in M$ if $\varphi^{\mathcal{M}}(\bar{a})=\hat{0}$. In this situation, we write $\mathcal{M} \models \varphi(\bar{a}) . T$ is satisfiable in $\mathcal{M}$, if $\mathcal{M} \models \psi$ for every $\psi \in T$. This is denoted by $\mathcal{M} \models T$.

2. For an $\mathcal{L}$-sentence $\varphi$, we say that $T$ entails $\varphi, T \models \varphi$, if for any $\mathcal{L}$ structure $\mathcal{M} \models T, \mathcal{M} \models \varphi$.

3. Likewise, $T$ strongly entails $\varphi, T \stackrel{s}{\models} \varphi$, if for any structure $\mathcal{M}, \varphi^{\mathcal{M}} \leq$ $\sup \left\{\psi^{\mathcal{M}}: \psi \in T\right\}$.

$T$ is called a satisfiable $\mathcal{L}$-theory if there is an $\mathcal{L}$-structure $\mathcal{M} \models T . T$ is a finitely satisfiable theory if every finite subset of $T$ is satisfiable.

\subsection{Axioms and the Proof System}

This subsection is devoted to introducing a complete proof system for $R G L^{*}$. The first part of axioms of $R G L^{*}$ are the axioms of propositional Gödel logic [10.

(G1) $(\varphi \rightarrow \psi) \rightarrow((\psi \rightarrow \chi) \rightarrow(\varphi \rightarrow \chi))$.

(G2) $(\varphi \wedge \psi) \rightarrow \varphi$.

(G3) $(\varphi \wedge \psi) \rightarrow(\psi \wedge \varphi)$.

(G4) $\varphi \rightarrow(\varphi \wedge \varphi)$.

(G5) $(\varphi \rightarrow(\psi \rightarrow \chi)) \leftrightarrow((\varphi \wedge \psi) \rightarrow \chi)$.

(G6) $((\varphi \rightarrow \psi) \rightarrow \chi) \rightarrow(((\psi \rightarrow \varphi) \rightarrow \chi) \rightarrow \chi)$.

(G7) $\overline{1} \rightarrow \varphi$.

The following axioms state the properties of the quantifiers $\forall$ and $\exists$.

$(\mathrm{G} \forall 1)(\forall x \varphi(x)) \rightarrow \varphi(t)(t$ substitutable for $x$ in $\varphi(x))$.

$(\mathrm{G} \forall 2)(\forall x(\psi \rightarrow \varphi(x))) \rightarrow(\psi \rightarrow(\forall x \varphi(x)))(x$ not free in $\psi)$.

$(\mathrm{G} \forall 3)(\forall x(\psi \vee \varphi(x))) \rightarrow(\psi \vee(\forall x \varphi(x)))(x$ not free in $\psi)$.

$(\mathrm{G} \exists 1) \varphi(t) \rightarrow(\exists x \varphi(x))(t$ substitutable for $x$ in $\varphi(x))$.

$(\mathrm{G} \exists 2)(\exists x(\psi \rightarrow \varphi(x))) \rightarrow(\psi \rightarrow(\exists x \varphi(x)))(x$ not free in $\psi)$. 
The inference rules are modus ponens and generalization:

$$
\frac{\varphi, \quad \varphi \rightarrow \psi}{\psi}, \quad \frac{\varphi}{\forall x \varphi} .
$$

The notion of proof is defined as usual. When an $\mathcal{L}$-theory $T$ proves an $\mathcal{L}$ sentence $\varphi$, we denote it by $T \vdash \varphi$. $T$ is called consistent if $T \nvdash \overline{1}$. Otherwise, it is inconsistent. The deduction theorem is stated as follows.

Theorem 2.5. $T \cup\{\varphi\} \vdash \psi$ iff $T \vdash \varphi \rightarrow \psi$.

Proof. See [10, Theorem 2.2.18]

An $\mathcal{L}$-theory $T$ is linear complete if for every pair of sentences $(\varphi, \psi)$, either $T \vdash \varphi \rightarrow \psi$ or $T \vdash \psi \rightarrow \varphi$.

Lemma 2.6. (Substitution lemma) Let $\Gamma$ be an $\mathcal{L}$-theory and $R, S$ be two nullary predicate symbols. Moreover, suppose $\Gamma(R, S) \vdash \chi(R, S)$. Then, for every $\mathcal{L}$ sentences $\varphi$ and $\psi, \Gamma(\varphi, \psi) \vdash \chi(\varphi, \psi)$.

Proof. Straightforward using the notion of proof.

Lemma 2.7. Suppose that $T$ is an $\mathcal{L}$-theory and $\varphi, \psi, \chi \in \operatorname{Sent}(\mathcal{L})$. Then,

$$
\begin{aligned}
& \text { (i) } \vdash \varphi \rightarrow(\psi \rightarrow \varphi) \text {. } \\
& \text { (ii) } \vdash \varphi \rightarrow(\psi \rightarrow(\varphi \wedge \psi)) \text {. } \\
& \text { (iii) } \vdash(\overline{0} \rightarrow \varphi) \rightarrow \varphi \text {. } \\
& \text { (iv) If } T \vdash \varphi \text { and } T \vdash \psi \text { then } T \vdash \varphi \wedge \psi \text {. } \\
& \text { (v) If } T \vdash \varphi \rightarrow \psi \text { and } T \vdash \psi \rightarrow \chi \text { then } T \vdash \varphi \rightarrow \chi \text {. } \\
& \text { (vi) } \vdash((\varphi \rightarrow \psi) \rightarrow(\psi \rightarrow \varphi)) \rightarrow(\psi \rightarrow \varphi) \text {. } \\
& \text { (vii) } \vdash \varphi \rightarrow(\varphi \vee \psi) \text {. } \\
& \text { (viii) } \vdash((\varphi \rightarrow \psi) \rightarrow \chi) \rightarrow((\psi \rightarrow \varphi) \vee \chi) .
\end{aligned}
$$

Proof. (i)-(vii) are obvious, [10, Chapter 2]. For (viii), by G6,

$$
\vdash((\varphi \rightarrow \psi) \rightarrow \chi) \rightarrow(((\psi \rightarrow \varphi) \rightarrow \chi) \rightarrow \chi) .
$$

So, by modus ponens,

$$
(\varphi \rightarrow \psi) \rightarrow \chi \vdash((\psi \rightarrow \varphi) \rightarrow \chi) \rightarrow \chi .
$$

On the other hand, by G1,

$$
\vdash((\varphi \rightarrow \psi) \rightarrow \chi) \rightarrow((\chi \rightarrow(\psi \rightarrow \varphi)) \rightarrow((\varphi \rightarrow \psi) \rightarrow(\psi \rightarrow \varphi))) .
$$

Therefore, by modus ponens,

$$
(\varphi \rightarrow \psi) \rightarrow \chi \vdash(\chi \rightarrow(\psi \rightarrow \varphi)) \rightarrow((\varphi \rightarrow \psi) \rightarrow(\psi \rightarrow \varphi)) .
$$


Now, by (vi) and using (v), we have

$$
(\varphi \rightarrow \psi) \rightarrow \chi \vdash(\chi \rightarrow(\psi \rightarrow \varphi)) \rightarrow(\psi \rightarrow \varphi) .
$$

Now, by (11), (2) and using (iv), we have

$$
(\varphi \rightarrow \psi) \rightarrow \chi \vdash((\chi \rightarrow(\psi \rightarrow \varphi)) \rightarrow(\psi \rightarrow \varphi)) \wedge(((\psi \rightarrow \varphi) \rightarrow \chi) \rightarrow \chi) .
$$

Thus, by definition of $\vee$,

$$
(\varphi \rightarrow \psi) \rightarrow \chi \vdash(\psi \rightarrow \varphi) \vee \chi
$$

Other axioms of $R G L^{*}$ called the book-keeping axioms, that is for any $\bar{r}, \bar{s} \in$ $\mathcal{A}$,

(RGL1) $\bar{r} \wedge \bar{s} \leftrightarrow \overline{\max \{r, s\}}$,

(RGL2) $\left\{\begin{array}{cc}\bar{r} \rightarrow \bar{s} & \text { if } r \geq s, \\ (\bar{r} \rightarrow \bar{s}) \leftrightarrow \bar{s} & \text { if } r<s,\end{array}\right.$

(RGL3) $\neg \neg \bar{r}$, for all $r<1$.

The following fact is used in the proof of the completeness theorem and its proof can be found in [10, Chapter 2].

Lemma 2.8. Let $T$ be an $\mathcal{L}$-theory.

1) Let $\sim$ be the relation on $\operatorname{Sent}(\mathcal{L})$ defined by

$$
\varphi \sim \psi \text { if and only if } T \vdash \varphi \leftrightarrow \psi \text {. }
$$

Then, $\sim$ is an equivalence relation.

2) Let $\mathcal{G}=\left\{[\varphi]_{T}: \varphi \in \operatorname{Sent}(\mathcal{L})\right\}$ be the set of equivalence classes of $\sim$. Define $\leq_{\mathcal{G}}$ on $\mathcal{G}$ by

$$
[\varphi]_{T} \leq_{\mathcal{G}}[\psi]_{T} \text { iff } T \vdash \psi \rightarrow \varphi .
$$

Then, $\leq_{\mathcal{G}}$ defines a partially order relation on $\mathcal{G}$.

3) For any $\bar{r} \in \mathcal{A}$, suppose $r^{\mathcal{G}}=[\bar{r}]_{T}$. Assume $\vee$, A and $\rightarrow$ are given by

$$
\begin{aligned}
& {[\varphi]_{T} \vee[\psi]_{T}=[\varphi \vee \psi]_{T},} \\
& {[\varphi]_{T} \wedge[\psi]_{T}=[\varphi \wedge \psi]_{T} \text {, }} \\
& {[\varphi]_{T} \rightarrow[\psi]_{T}=[\varphi \rightarrow \psi]_{T},}
\end{aligned}
$$

respectively. Then, $\left(\mathcal{G}, \vee, A, \rightarrow,[\overline{0}]_{T},[\overline{1}]_{T}\right)$ is a resituated lattice with the largest element $[\overline{1}]_{T}$ and the least element $[\overline{0}]_{T}$ where $\mathcal{A}$ and $\rightarrow$ form an adjoint pair, i.e., for all $a, b, c \in \mathcal{G}$,

$$
a \wedge b \geq_{\mathcal{G}} c \text { iff } a \geq_{\mathcal{G}} b \rightarrow c .
$$


In some literatures, $\mathcal{G}$ is called a $G$-algebra and the structure $(\mathcal{G}, \vee, \wedge, \rightarrow$ ,$\left.\left\{[\bar{r}]_{T}: r \in[0,1]_{\mathbb{Q}}\right\}\right)$ is called an $R G L$-algebra.

The following crucial definition is needed for the completeness of $R G L^{*}$.

Definition 2.9. An $\mathcal{L}$-theory $T$ is called strongly consistent if $T \nvdash \bar{r}$ for every $r>0$.

Clearly, if $\mathrm{T}$ is a strongly consistent theory then for each $r>0$, we have $[\bar{r}]_{T}>[\overline{0}]_{T}$. Furthermore, by RGL3, since for every $r<1$, we have $[\bar{r}]_{T}<[\overline{1}]_{T}$. Hence, the function $r \rightarrow[\bar{r}]_{T}$ defines an order isomorphism of $[0,1]_{\mathbb{Q}}$ onto $\left\{r^{\mathcal{G}}\right.$ : $\left.r \in[0,1]_{\mathbb{Q}}\right\}$.

Remark 2.10. Using the usual proof of soundness theorem, one may show that if $T \vdash \varphi$ then $T \models \varphi$ for every $\mathcal{L}$-theory $T$ and $\mathcal{L}$-sentence $\varphi$.

\subsection{Completeness of $R G L^{*}$}

In this subsection, it is shown that any strongly consistent theory is satisfiable. Consequently, the compactness theorem is proved. Our proof is based on Henkin construction. First, some preliminary lemmas are given.

Lemma 2.11. Let $T$ be a strongly consistent $\mathcal{L}$-theory in $R G L^{*}$ and $\varphi, \psi \in$ $\operatorname{Sent}(\mathcal{L})$. Then, either $T \cup\{\varphi \rightarrow \psi\}$ or $T \cup\{\psi \rightarrow \varphi\}$ are strongly consistent.

Proof. Suppose on the contrary that both $T \cup\{\varphi \rightarrow \psi\}$ and $T \cup\{\psi \rightarrow \varphi\}$ are not strongly consistent. So, there are $r, s>0$ such that $T \cup\{\varphi \rightarrow \psi\} \vdash \bar{r}$ and $T \cup\{\psi \rightarrow \varphi\} \vdash \bar{s}$. Thus, by the deduction theorem, $T \vdash(\varphi \rightarrow \psi) \rightarrow \bar{r}$ and $T \vdash(\psi \rightarrow \varphi) \rightarrow \bar{s}$. Without loss of generality, we may suppose that $\min \{r, s\}=r$. Therefore, by RGL2, $\vdash \bar{s} \rightarrow \bar{r}$. Now, using Lemma 2.7(v), we have $T \vdash(\varphi \rightarrow \psi) \rightarrow \bar{r}$ and $T \vdash(\psi \rightarrow \varphi) \rightarrow \bar{r}$. Hence, by G6, we have $T \vdash \bar{r}$, a contradiction.

Next, the notion of maximally strongly consistent theory is defined.

Definition 2.12. A strongly consistent theory $T$ is called maximally strongly consistent if it can not be properly included in any strongly consistent theory, i.e., for any strongly consistent theory $\Sigma$, if $T \subseteq \Sigma$ then $T=\Sigma$.

Lemma 2.13. Let $T$ be a strongly consistent $\mathcal{L}$-theory. $T$ is maximally strongly consistent if and only if

1. for all $\varphi, \psi \in \operatorname{Sent}(\mathcal{L})$, either $\varphi \rightarrow \psi \in T$ or $\psi \rightarrow \varphi \in T$,

2. if $\varphi \in \operatorname{Sent}(\mathcal{L})$ and $T \vdash \bar{r} \rightarrow \varphi$ for all $r>0$, then $\varphi \in T$.

Proof. (1) is easily derived from Lemma 2.11, For (2), let $T \vdash \bar{r} \rightarrow \varphi$ for all $r>0$. We show that $T \cup\{\varphi\}$ is strongly consistent. Suppose on the contrary, $T \cup\{\varphi\}$ is not strongly consistent. Then, there is $k>0$ such that $T \cup\{\varphi\} \vdash k$. So, by the deduction theorem, $T \vdash \varphi \rightarrow \bar{k}$. Hence, using Lemma 2.7(v), $T \vdash \bar{r} \rightarrow \bar{k}$ for all $r>0$. In particular, if we take $r<k$ we get a contradiction.

Conversely, let (1) and (2) hold and $\Sigma$ be a strongly consistent theory containing $T$. If $\varphi \notin T$ then it implies there exists $r>0$ such that $T \nvdash \bar{r} \rightarrow \varphi$. So, $\varphi \rightarrow \bar{r} \in T$ and therefore, $\varphi \rightarrow \bar{r} \in \Sigma$. Thus, $\varphi \notin \Sigma$. 
Now, an easy application of Zorn's lemma ensures the existence of a maximally strongly consistent extension of every strongly consistent theory. Hence, the following lemma is established.

Lemma 2.14. There exists a maximally strongly consistent extension of every strongly consistent theory.

Lemma 2.15. Let $T$ be a maximally strongly consistent theory and $T \vdash \varphi \vee \psi$. Then, either $T \vdash \varphi$ or $T \vdash \psi$.

Proof. Proof is straightforward.

Now, the definition of a Henkin theory is given.

Definition 2.16. An $\mathcal{L}$-theory $T$ is Henkin if whenever $T \nvdash \forall x \varphi(x)$, there is a constant symbol $c \in \mathcal{L}$ such that $T \nvdash \varphi(c)$.

Theorem 2.17. Assume $T$ is a strongly consistent $\mathcal{L}$-theory. Then, there exist an extension $\hat{\mathcal{L}}$ of $\mathcal{L}$ and a maximally strongly consistent Henkin $\hat{\mathcal{L}}$-theory $\hat{T}$ containing $T$.

Proof. Let $\mathcal{L}_{0}=\mathcal{L}$ and $T_{0}=T$. Suppose that $\mathcal{L}_{n}$ and $T_{n}$ are constructed such that $T_{n}$ is a strongly consistent $\mathcal{L}_{n}$-theory containing $T$. Let $F_{0}=\operatorname{Form}\left(\mathcal{L}_{0}\right)$. For $n>0$, set $F_{n}=\operatorname{Form}\left(\mathcal{L}_{n}\right) \backslash \operatorname{Form}\left(\mathcal{L}_{n-1}\right)$. Define

$$
\mathcal{L}_{n+1}=\mathcal{L}_{n} \cup\left\{c_{\varphi(x), r, s}: \varphi(x) \in F_{n}, r>s>0\right\}
$$

and take a maximally strongly consistent $\mathcal{L}_{n}$-theory $\hat{T}_{n}$ containing $T_{n}$. Take

$$
T_{n+1}=\hat{T}_{n} \cup\left\{(\bar{r} \rightarrow \forall x \varphi(x)) \vee\left(\varphi\left(c_{\varphi(x), r, s}\right) \rightarrow \bar{s}\right): \varphi(x) \in F_{n}, r>s>0\right\} .
$$

Claim 1. $T_{n+1}$ is strongly consistent.

Proof of claim 1.

Suppose not. Therefore, $T_{n+1} \vdash \bar{k}$ for some $k>0$. So, there is a finite subset $\Sigma$ of $\left\{(\bar{r} \rightarrow \forall x \varphi(x)) \vee\left(\varphi\left(c_{\varphi(x), r, s}\right) \rightarrow \bar{s}\right): \varphi(x) \in F_{n}, r>s>0\right\}$ such that $\hat{T}_{n} \cup \Sigma \vdash \bar{k}$. Take $\Sigma$ to be minimal. For $\theta_{\varphi(x), r, s} \in \Sigma$, let $\Gamma=\Sigma \backslash\left\{\theta_{\varphi(x), r, s}\right\}$. Now, by showing that $\hat{T}_{n} \cup \Gamma \vdash \bar{k}$, we get a contradiction. i.e.,

Since $\hat{T}_{n} \cup \Gamma \cup\left\{\theta_{\varphi(x), r, s}\right\} \vdash \bar{k}$, by the deduction theorem, $\hat{T}_{n} \cup \Gamma \vdash \theta_{\varphi(x), r, s} \rightarrow \bar{k}$,

$$
\hat{T}_{n} \cup \Gamma \vdash\left((\bar{r} \rightarrow \forall x \varphi(x)) \vee\left(\varphi\left(c_{\varphi(x), r, s}\right) \rightarrow \bar{s}\right)\right) \rightarrow \bar{k} .
$$

Therefore, by Lemma 2.7](vii),

$$
\hat{T}_{n} \cup \Gamma \vdash(\bar{r} \rightarrow \forall x \varphi(x)) \rightarrow \bar{k}
$$

and

$$
\hat{T}_{n} \cup \Gamma \vdash\left(\varphi\left(c_{\varphi(x), r, s}\right) \rightarrow \bar{s}\right) \rightarrow \bar{k}
$$

Since $\vdash \bar{s} \rightarrow\left(\varphi\left(c_{\varphi(x), r, s}\right) \rightarrow \bar{s}\right)$, (4) implies $\hat{T}_{n} \cup \Gamma \vdash \bar{s} \rightarrow \bar{k}$. Now, since $r>s$, by RGL2 and Lemma 2.7(v),

$$
\hat{T}_{n} \cup \Gamma \vdash \bar{r} \rightarrow \bar{k} .
$$


As $\hat{T}_{n} \cup \Gamma \nvdash \bar{k}$, it follows from (3) that $\hat{T}_{n} \cup \Gamma \nvdash \bar{r} \rightarrow \forall x \varphi(x)$. But, since $\hat{T}_{n}$ is a maximally strongly consistent $\mathcal{L}_{n}$-theory and $(\bar{r} \rightarrow \forall x \varphi(x)) \in \operatorname{Sent}\left(\mathcal{L}_{n}\right)$,

$$
\hat{T}_{n} \cup \Gamma \vdash \forall x \varphi(x) \rightarrow \bar{r} .
$$

On the other hand, by (4) and Lemma 2.7(vii),

$$
\hat{T}_{n} \cup \Gamma \vdash \bar{k} \vee\left(\bar{s} \rightarrow \varphi\left(c_{\varphi(x), r, s}\right)\right) .
$$

So, $c_{\varphi(x), r, s} \notin \mathcal{L}_{n}$ implies that

$$
\hat{T}_{n} \cup \Gamma \vdash \forall x(\bar{k} \vee(\bar{s} \rightarrow \varphi(x))) .
$$

Therefore, by $\mathrm{G} \forall 3$,

$$
\hat{T}_{n} \cup \Gamma \vdash \bar{k} \vee \forall x(\bar{s} \rightarrow \varphi(x)) .
$$

Now, since $\hat{T}_{n} \cup \Gamma \nvdash \bar{k}$ and $\hat{T}_{n}$ is maximally strongly consistent $\mathcal{L}_{n}$-theory, by Lemma 2.15. $\hat{T}_{n} \cup \Gamma \vdash \forall x(\bar{s} \rightarrow \varphi(x))$. Moreover, by G $\forall 2$,

$$
\hat{T}_{n} \cup \Gamma \vdash \bar{s} \rightarrow \forall x \varphi(x) .
$$

(6) and Lemma 2.7(v) imply that $\hat{T}_{n} \cup \Gamma \vdash \bar{s} \rightarrow \bar{r}$. Furthermore, as $r>s$, by RGL2 and (5),

$$
\hat{T}_{n} \cup \Gamma \vdash \bar{k},
$$

a contradiction.

Now, using the above claim for each $n \geq 0$, one may inductively define a language $\mathcal{L}_{n}$ and a strongly consistent $\mathcal{L}_{n}$-theory $T_{n}$. Let $\hat{\mathcal{L}}=\bigcup_{n<\omega} \mathcal{L}_{n}$ and $\hat{T}$ be a maximally strongly consistent $\hat{\mathcal{L}}$-theory containing $\bigcup_{n<\omega} T_{n}$. We show that $\hat{T}$ is also a Henkin $\hat{\mathcal{L}}$-theory.

Clearly, as $\bigcup_{n<\omega} T_{n}$ is strongly consistent, $\hat{T}$ exists. To show that $\hat{T}$ is Henkin, let $\hat{T} \nvdash \forall x \varphi(x)$ for some $\varphi(x) \in \operatorname{Form}(\hat{\mathcal{L}})$. Now, since $\hat{T}$ is maximally strongly consistent there exists $r>0$ such that $\hat{T} \nvdash \bar{r} \rightarrow \forall x \varphi(x)$. But, $(\bar{r} \rightarrow \forall x \varphi(x)) \vee$ $\left(\varphi\left(c_{\varphi(x), r, s}\right) \rightarrow \bar{s}\right) \in \hat{T}$ implies $\hat{T} \vdash \varphi\left(c_{\varphi(x), r, s}\right) \rightarrow \bar{s}$ and so, $\hat{T} \nvdash \varphi\left(c_{\varphi(x), r, s}\right)$.

The following technical lemma is needed for Theorem 2.19

Lemma 2.18. Let $T$ be an $\mathcal{L}$-theory. Set

- $S=\{\psi \in \operatorname{Sent}(\mathcal{L})$ : there exists rational numbers $r, s>0$ such that $T \vdash$ $(\bar{s} \rightarrow \psi) \wedge(\psi \rightarrow \bar{r})\}$

- $\mathcal{L}^{\prime}=\mathcal{L} \cup\left\{\alpha_{\psi}, \beta_{\psi}: \psi \in S\right\} \cup\{\gamma\}$ where $\alpha_{\psi}, \beta_{\psi}$ and $\gamma$ are some new nullary predicate symbols.

- $T_{\psi}=T \cup\left\{\alpha_{\psi} \Rightarrow \psi, \psi \Rightarrow \beta_{\psi}\right\} \cup\left\{\bar{s} \rightarrow \alpha_{\psi}: T \vdash \bar{s} \Rightarrow \psi\right\} \cup\left\{\beta_{\psi} \rightarrow \bar{r}: T \vdash\right.$ $\psi \Rightarrow \bar{r}\}$.

- $T_{1}=T \cup\{\psi \Rightarrow \gamma\} \cup\{\gamma \rightarrow \bar{r}: r<1\}$. 
- $T^{\prime}=T_{1} \cup \bigcup_{\psi \in S} T_{\psi}$.

If $T$ is strongly consistent then so is $T^{\prime}$.

Proof. We only show that $T_{\psi}$ is strongly consistent. If not, then there exist a finite subset $\Delta$ of $T$ and $\bar{k}$ such that,

$$
\begin{aligned}
\Delta \cup\left\{\alpha_{\psi} \Rightarrow \psi, \psi \Rightarrow \beta_{\psi}\right\} \cup \quad & \quad\left\{\bar{s}_{i} \rightarrow \alpha_{\psi}: T \vdash \bar{s}_{i} \Rightarrow \psi, 1 \leq i \leq n\right\} \\
\cup \quad & \left\{\beta_{\psi} \rightarrow \bar{r}_{j}: T \vdash \psi \Rightarrow \bar{r}_{j}, 1 \leq j \leq m\right\} \vdash \bar{k} .
\end{aligned}
$$

We may suppose both sequences $\left\{s_{i}\right\}_{i=1}^{n}$ and $\left\{r_{j}\right\}_{j=1}^{m}$ are increasing. So, we have

$$
T \vdash\left(\bar{s}_{1} \Rightarrow \psi\right) \wedge\left(\psi \Rightarrow \bar{r}_{m}\right) \text { and } r_{1} \leq \ldots \leq r_{m}<s_{1} \leq \ldots \leq s_{n} .
$$

Now, using the substitution Lemma 2.6, one can replace $\alpha_{\psi}$ by $\bar{s}_{1}$ and $\beta_{\psi}$ by $\bar{r}_{m}$ and conclude:

$$
\begin{aligned}
\Delta \cup\left\{\bar{s}_{1} \Rightarrow \psi, \psi \Rightarrow \bar{r}_{m}\right\} \quad & \cup \quad\left\{\bar{s}_{i} \rightarrow \bar{s}_{1}: T \vdash \bar{s}_{i} \Rightarrow \psi, 1 \leq i \leq n\right\} \\
\cup \quad & \left\{\bar{r}_{m} \rightarrow \bar{r}_{j}: T \vdash \psi \Rightarrow \bar{r}_{j}, 1 \leq j \leq m\right\} \vdash \bar{k} .
\end{aligned}
$$

On the other hand,

$$
\begin{aligned}
T & \vdash \quad\left(\bar{s}_{1} \Rightarrow \psi\right) \wedge\left(\psi \Rightarrow \bar{r}_{m}\right), \\
& \vdash \quad\left(\bar{s}_{i} \rightarrow \bar{s}_{1}\right), \quad \text { for all } 1 \leq i \leq n, \\
& \vdash \quad\left(\bar{r}_{m} \rightarrow \bar{r}_{j}\right), \quad \text { for all } 1 \leq j \leq m .
\end{aligned}
$$

Thus, $T \vdash \bar{k}$, a contradiction.

Theorem 2.19. Let $\Sigma$ be a maximally strongly consistent $\mathcal{L}$-theory and $\mathcal{G}$ be the RGL-algebra of classes of $\Sigma$-equivalent sentences introduced in Lemma 2.8. Then, there is a continuous order preserving map $g$ from $\mathcal{G}$ into $\mathbb{I}$ (that is all suprema and infima preserved by the map $g$ ).

Proof. For any $a \in \mathcal{G}$, set

$$
\operatorname{st}(a)=\inf \left\{r \in[0,1]_{\mathbb{Q}}: a \leq_{\mathcal{G}}[\bar{r}]_{\Sigma}\right\} \in[0,1] .
$$

Let $\langle a\rangle_{\mathcal{G}}=\{b \in \mathcal{G}: s t(b)=s t(a)\}$. Note that if $b \in\langle a\rangle_{\mathcal{G}}$ then, for any $r>0$,

$$
\Sigma \vdash \bar{r} \rightarrow a \text { iff } \Sigma \vdash \bar{r} \rightarrow b \text {. }
$$

To construct $g$, for any $a \in \mathcal{G}$, we define $g_{a}$ from $\langle a\rangle_{\mathcal{G}}$ to

$$
\mathbb{I}_{a}=\{x \in \mathbb{I}:(\operatorname{st}(a), 0) \leq x \leq(\operatorname{st}(a), 1)\}
$$

and let $g=\bigcup_{a \in \mathcal{G}} g_{a}$.

Lemma 2.18 shows that one may extend the language $\mathcal{L}$ so that for any $[\overline{0}]_{\Sigma}<\mathcal{G}$ $a,\langle a\rangle_{\mathcal{G}}$ has at least two distinct elements, and furthermore, for any rational number $0<r<1$, there are two distinct elements $a, b \in\left\langle[\bar{r}]_{\Sigma}\right\rangle_{\mathcal{G}}$ such that $a<_{\mathcal{G}}[\bar{r}]_{\Sigma}<_{\mathcal{G}} b$. Thus, since $\langle a\rangle_{\mathcal{G}}$ is a countable set by the same way as [10, Lemma 5.3.1], for any $[\overline{0}]_{\Sigma}<_{\mathcal{G}} a$, there is a continuous order preserving map $g_{a}$ of $\langle a\rangle_{\mathcal{G}}$ into $\mathbb{I}_{a}$ such that 
1) If $\langle a\rangle_{\mathcal{G}}$ has minimum (resp. maximum) $\alpha$, then $g_{a}(\alpha)$ is the minimum (resp. maximum) of $\mathbb{I}_{a}$,

2) for each rational number $r \in[0,1], g_{r}\left([\bar{r}]_{\Sigma}\right)=\hat{r}$.

In the light of the above Lemma 2.18, (1) and (2) simultaneously hold.

The proof is completed, if we show that $g=\bigcup_{a \in \mathcal{G}} g_{a}$ is a continuous order preserving function.

Linear completeness of $\Sigma$ implies that whenever $\langle a\rangle_{\mathcal{G}}\left\langle\langle b\rangle_{\mathcal{G}}\right.$, there is $r>0$ such that $a<_{\mathcal{G}}[\bar{r}]_{\Sigma}<_{\mathcal{G}} b$ and whence, $g$ is an order preserving function. To prove the continuity of $g$, let $A \subseteq \mathcal{G}$ and $\sup A=\alpha$ and $\inf A=\beta$. We have to verify that $\sup g(A)=g(\alpha)$ and $\inf g(A)=g(\beta)$, respectively. We only show that $\sup g(A)=g(\alpha)$ and the other case is similar. The proof is divided in two different cases:

a) $\alpha \in\langle a\rangle_{\mathcal{G}}$ for some $a \in \mathcal{G}$, and furthermore, there is some $e \neq \alpha$ in $A$ such that $e \in\langle a\rangle_{\mathcal{G}}$. In this case, continuity of $g_{a}$ implies that

$$
\begin{aligned}
\sup g(A) & =\sup \{g(b): b \in A\}=\sup \left\{g(b): b \in A \cap\langle a\rangle_{\mathcal{G}}\right\} \\
& =\sup \left\{g_{a}(b): b \in A \cap\langle a\rangle_{\mathcal{G}}\right\}=g_{a}\left(\sup \left\{b: b \in A \cap\langle a\rangle_{\mathcal{G}}\right\}\right)=g_{a}(\alpha) .
\end{aligned}
$$

b) $\alpha \in\langle a\rangle_{\mathcal{G}}$ and there is no element of $A$ in $\langle a\rangle_{\mathcal{G}}$. Subsequently, we have two subcases:

b1) $g(\alpha), \sup g(A) \in \mathbb{I}_{a}$. Since $b \notin\langle a\rangle_{\mathcal{G}}$ for each $b \in A$, it follows $\alpha=$ $\min \langle a\rangle_{\mathcal{G}}$. Thus, $g(\alpha)=g_{a}(\alpha)=\min \left(\mathbb{I}_{a}\right)$ by the condition (1) above. On the other hand, as $\sup g(A) \in \mathbb{I}_{a}$, it implies $\sup g(A)=\min \left(\mathbb{I}_{a}\right)$. Therefore, $\sup g(A)=g(\alpha)$.

b2) $g(\alpha) \in \mathbb{I}_{a}$, but $\sup g(A) \notin \mathbb{I}_{a}$. This means $\sup g(A)<g(\alpha)$. So, there is a rational number $r<s t(a)$ such that $\sup g(A)<\hat{r}<g(\alpha)$. Therefore, $\sup g(A)<g\left([\bar{r}]_{\Sigma}\right)$. But, this contradicts the fact that $\alpha$ is the least upper bound of $A$.

Next, the completeness theorem is established for $R G L^{*}$.

Theorem 2.20. (Completeness of $R G L^{*}$ ) In $R G L^{*}$, any strongly consistent theory is satisfiable.

Proof. Let $T$ be a strongly consistent $\mathcal{L}$-theory. By Theorem 2.17 there is a first order language $\hat{\mathcal{L}}$ containing $\mathcal{L}$ and a maximally strongly consistent Henkin $\hat{\mathcal{L}}$-theory $\Sigma$ containing $T$. Let $\overline{\mathcal{G}}$ be the $R G L$-algebra of classes of $\Sigma$-equivalent sentences introduced in Lemma 2.8. Since $\Sigma$ is linear complete, $\mathcal{G}$ is a linear ordered set. Now, in the light of Theorem [2.19, there is a continuous order preserving map from $\mathcal{G}$ into $\mathbb{I}$. Therefore, one can define an $\mathcal{L}$-structure $\mathcal{M}$ as follows.

a) The universe of $\mathcal{M}$ is the set of all closed $\hat{\mathcal{L}}$-terms.

b) For each n-ary function symbol $f$, define $f^{\mathcal{M}}: M^{n} \rightarrow M$ as

$$
f^{\mathcal{M}}\left(t_{1}, \ldots, t_{n}\right)=f\left(t_{1}, \ldots, t_{n}\right) .
$$


c) For each n-ary predicate symbol $P$, define $P^{\mathcal{M}}: M^{n} \rightarrow \mathbb{I}$ as

$$
P^{\mathcal{M}}\left(t_{1}, \ldots, t_{n}\right)=g\left(\left[P\left(t_{1}, \ldots, t_{n}\right)\right]_{\Sigma}\right) .
$$

A straightforward induction on the complexity of formulas shows that $\varphi^{\mathcal{M}}=$ $g\left([\varphi]_{\Sigma}\right)$ for any $\varphi \in \operatorname{Sent}(\hat{\mathcal{L}})$. In particular, for any $\varphi \in \Sigma, \varphi^{\mathcal{M}}=\hat{0}$. Since $T \subseteq \Sigma$, it follows that $\mathcal{M}$ is a model of $T$ and the proof is complete.

Corollary 2.21. (Compactness theorem for $R G L^{*}$ ) Every finitely satisfiable $\mathcal{L}$-theory is satisfiable.

Also, one may study the strong completeness with respect to the strong entailment.

\subsection{Completeness with respect to the strong entailment}

In this subsection, we investigate the relationship between strong entailment and deduction. First, we prove the weak completeness of $R G L^{*}$. A different but similar proof can be found in [9, Theorem 4.7].

Lemma 2.22. (Weak completeness theorem) For every sentence $\varphi$, if $\models \varphi$ then $\vdash \varphi$.

Proof. Let $\nvdash \varphi$ in $R G L^{*}$ and $\bar{r}_{1}, \ldots, \bar{r}_{n} \in \operatorname{Sub}(\varphi)$. Let $G\left(r_{1}, \ldots, r_{n}\right)$ be the Gödel logic enriched with finitely many nullary predicate $\bar{r}_{1}, \ldots, \bar{r}_{n}$ introduced in 10. Note that any sentence which is derivable in $G\left(r_{1}, \ldots, r_{n}\right)$ can also be derived in $R G L^{*}$. The set $A x\left(r_{1}, \ldots, r_{n}\right)$ of book-keeping axioms of $G\left(r_{1}, \ldots, r_{n}\right)$ can also be viewed as an $\mathcal{L} \cup\left\{\bar{r}_{1}, \ldots, \bar{r}_{n}\right\}$-theory in first-order Gödel logic. This means that $\bar{r}_{1}, \ldots, \bar{r}_{n}$ are treated as nullary predicates. Hence, $A x\left(r_{1}, \ldots, r_{n}\right) \nvdash \varphi$ in (standard) first-order Gödel logic. Now, on the basis of standard completeness theorem of Gödel logic, there is a countable structure $\mathcal{M}$, whose truth values take place in $[0,1]$ such that $\varphi^{\mathcal{M}}>0$. Note that for $1 \leq i \leq n, \bar{r}_{i}$ may not be interpreted by $r_{i}$. Let

$$
X=\left\{\psi^{\mathcal{M}}(\bar{a}) \mid \psi(\bar{x}) \in \operatorname{Sub}(\varphi), \bar{a} \in M^{n}\right\} .
$$

Take $\alpha=\max \left\{r>0 \mid \bar{r}^{\mathcal{M}}=0, \bar{r} \in \operatorname{Sub}(\varphi)\right\} \cup\{\overline{0}\}$. Since $X$ is countable there is a continuous order-preserving map $g: X \rightarrow[\alpha, 1]^{2}$ such that $g(\bar{r})=\hat{r}$, for every $r>\alpha$ and $g\left(\overline{0}^{\mathcal{M}}\right)=\hat{\alpha}$. We construct an $\mathcal{L}$-structure $\mathcal{N}$ as follows. Let the universe of $\mathcal{N}$ as well as the interpretations of function and constant symbols remain the same as the structure $\mathcal{M}$. For every predicate symbol $P$ and $\bar{a} \in M$, define

$$
P^{\mathcal{N}}(\bar{a})=\left\{\begin{array}{cc}
g\left(P^{\mathcal{M}}(\bar{a})\right) & P(\bar{x}) \in \operatorname{Sub}(\varphi), \\
\hat{0} & \text { o.w. }
\end{array}\right.
$$

We claim that for every $\psi(\bar{x}) \in \operatorname{Sub}(\varphi)$ and $\bar{a} \in M^{n}$,

1. if $\psi^{\mathcal{M}}(\bar{a})>0$ then $\psi^{\mathcal{N}}(\bar{a})=g\left(\psi^{\mathcal{M}}(\bar{a})\right)>\hat{\alpha}$, and

2. if $\psi^{\mathcal{M}}(\bar{a})=0$ then $\psi^{\mathcal{N}}(\bar{a}) \leq \hat{\alpha}$.

The claim can be proved by induction.

1. For atomic formulas, the connectives $\wedge, \vee$, and the quantifier $\forall$, the induction is routine. 
2. Let $\psi(\bar{x})=\psi_{1}(\bar{x}) \rightarrow \psi_{2}(\bar{x})$.

If $\left(\psi_{1} \rightarrow \psi_{2}\right)^{\mathcal{M}}(\bar{a})>0$ then $\psi_{1}^{\mathcal{M}}(\bar{a})<\psi_{2}^{\mathcal{M}}(\bar{a})$ and $\psi_{2}^{\mathcal{M}}(\bar{a})>0$.

- If $\psi_{1}^{\mathcal{M}}(\bar{a})=0$ then by induction hypotheses, $\psi_{1}^{\mathcal{N}}(\bar{a}) \leq \hat{\alpha}$ and $\psi_{2}^{\mathcal{N}}(\bar{a})=$ $g\left(\psi_{2}^{\mathcal{M}}(\bar{a})\right)>\hat{\alpha}$. Therefore, $\psi_{1}^{\mathcal{N}}(\bar{a})<\psi_{2}^{\mathcal{N}}(\bar{a})$ and $\left(\psi_{1} \rightarrow \psi_{2}\right)^{\mathcal{N}}(\bar{a})=$ $\psi_{2}^{\mathcal{N}}(\bar{a})>\hat{\alpha}$.

- If $0<\psi_{1}^{\mathcal{M}}(\bar{a})<\psi_{2}^{\mathcal{M}}(\bar{a})$ then $\hat{\alpha}<\psi_{1}^{\mathcal{N}}(\bar{a})=g\left(\psi_{1}^{\mathcal{M}}(\bar{a})\right)<\psi_{2}^{\mathcal{N}}(\bar{a})=$ $g\left(\psi_{2}^{\mathcal{M}}(\bar{a})\right)$. So, $\left(\psi_{1} \rightarrow \psi_{2}\right)^{\mathcal{N}}(\bar{a})=\psi_{2}^{\mathcal{N}}(\bar{a})>\hat{\alpha}$.

If $\left(\psi_{1} \rightarrow \psi_{2}\right)^{\mathcal{M}}(\bar{a})=0$ then $\psi_{1}^{\mathcal{M}}(\bar{a}) \geq \psi_{2}^{\mathcal{M}}(\bar{a})$. Suppose on the contrary, $\left(\psi_{1} \rightarrow \psi_{2}\right)^{\mathcal{N}}(\bar{a})>\hat{\alpha}$. Then, $\psi_{1}^{\mathcal{N}}(\bar{a})<\psi_{2}^{\mathcal{N}}(\bar{a})$ and $\psi_{2}^{\mathcal{N}}(\bar{a})>\hat{\alpha}$. This means $\psi_{2}^{\mathcal{N}}(\bar{a})=g\left(\psi_{2}^{\mathcal{M}}(\bar{a})\right)>g(0)=\hat{\alpha}$. Now, since $g$ is monotone $\psi_{2}^{\mathcal{M}}(\bar{a})>0$. On the other hand, $\psi_{1}^{\mathcal{M}}(\bar{a}) \geq \psi_{2}^{\mathcal{M}}(\bar{a})>0$ implies that $\hat{\alpha}<\psi_{2}^{\mathcal{N}}(\bar{a})=$ $g\left(\psi_{2}^{\mathcal{M}}(\bar{a})\right) \leq \psi_{1}^{\mathcal{N}}(\bar{a})=g\left(\psi_{1}^{\mathcal{M}}(\bar{a})\right)$, a contradiction.

3. Let $\psi(\bar{x})=\exists y \psi_{1}(y, \bar{x})$.

If $\left(\exists y \psi_{1}(y, \bar{b})\right)^{\mathcal{M}}>0$ then $\inf _{a \in M} \psi_{1}^{\mathcal{M}}(a, \bar{b})>0$. This implies that $\psi_{1}^{\mathcal{M}}(a, \bar{b})>0$ for every $a \in M$. So, by induction hypothesis, $\psi_{1}^{\mathcal{N}}(a, \bar{b})=$ $g\left(\psi_{1}^{\mathcal{M}}(a, \bar{b})\right)>\hat{\alpha}$ for every $a \in M$. By the continuity of $g$, we have

$$
\begin{aligned}
\left(\exists y \psi_{1}(y, \bar{b})\right)^{\mathcal{N}} & =\inf _{a \in N} \psi_{1}^{\mathcal{N}}(a, \bar{b}) \\
& =\inf _{a \in M} g\left(\psi_{1}^{\mathcal{M}}(a, \bar{b})\right) \\
& =g\left(\inf _{a \in M} \psi_{1}^{\mathcal{M}}(a, \bar{b})\right)>\hat{\alpha} .
\end{aligned}
$$

Now, suppose $\left(\exists y \psi_{1}(y, \bar{b})\right)^{\mathcal{M}}=0$. Then, $\inf _{a \in M} \psi_{1}^{\mathcal{M}}(a, \bar{b})=0$.

- If there is an element $a \in M$ such that $\psi_{1}^{\mathcal{M}}(a, \bar{b})=0$ then by induction hypothesis, $\psi_{1}^{\mathcal{N}}(a, \bar{b}) \leq \hat{\alpha}$. So, $\left(\exists y \psi_{1}(y, \bar{b})\right)^{\mathcal{N}}=\inf _{a \in N} \psi_{1}^{\mathcal{N}}(a, \bar{b}) \leq$ $\hat{\alpha}$.

- If for every $a \in M, \psi_{1}^{\mathcal{M}}(a, \bar{b})>0$ then for every $a \in N, \psi_{1}^{\mathcal{N}}(a, \bar{b})=$ $g\left(\psi_{1}^{\mathcal{M}}(a, \bar{b})\right)>\hat{\alpha}$. Hence,

$$
\begin{aligned}
\left(\exists y \psi_{1}(x, \bar{b})\right)^{\mathcal{N}} & =\inf _{a \in N} \psi_{1}^{\mathcal{N}}(a, \bar{b}) \\
& =\inf _{a \in M} g\left(\psi_{1}^{\mathcal{M}}(a, \bar{b})\right) \\
& =g\left(\inf _{a \in M} \psi_{1}^{\mathcal{M}}(a, \bar{b})\right)=g(0)=\hat{\alpha} .
\end{aligned}
$$

The following theorem establishes some connections between the strong entailment and deduction.

Theorem 2.23. Let $\Sigma$ be an $\mathcal{L}$-theory and $\varphi$ be an $\mathcal{L}$-sentence.

1) if $\Sigma$ is finite then, $\Sigma \models \stackrel{s}{=} \varphi$ iff $\Sigma \vdash \varphi$.

2) $\Sigma \stackrel{s}{=} \varphi$ if and only if $\Sigma \vdash \overline{n^{-1}} \rightarrow \varphi$ for all $n \in \mathbb{N}$.

Proof. (1). Let $\Sigma=\left\{\varphi_{1}, \ldots, \varphi_{n}\right\}$. If $\left\{\varphi_{1}, \ldots, \varphi_{n}\right\} \stackrel{s}{=} \varphi$ then for all $\mathcal{L}$-structures $\mathcal{M}$, 


$$
\max \left\{\varphi_{1}^{\mathcal{M}}, \ldots, \varphi_{n}^{\mathcal{M}}\right\} \geq \varphi^{\mathcal{M}} .
$$

So, for all $\mathcal{L}$-structures $\mathcal{M},\left(\varphi_{1} \wedge \cdots \wedge \varphi_{n}\right)^{\mathcal{M}} \geq \varphi^{\mathcal{M}}$ and $\left(\varphi_{1} \wedge \cdots \wedge \varphi_{n} \rightarrow \varphi\right)^{\mathcal{M}}=0$. Therefore, $\models \varphi_{1} \wedge \cdots \wedge \varphi_{n} \rightarrow \varphi$. By Lemma 2.22, $\vdash \varphi_{1} \wedge \cdots \wedge \varphi_{n} \rightarrow \varphi$. So, by the deduction theorem, $\varphi_{1} \wedge \cdots \wedge \varphi_{n} \vdash \varphi$. Hence, $\Sigma \vdash \varphi$.

The other direction easily follows from the soundness theorem.

(2). We first show that whenever $\left.\Sigma\right|^{s} \varphi$, for every $n \in \mathbb{N}$, there is a finite subset $\Sigma_{0}$ of $\Sigma$ such that $\Sigma_{0} \triangleq \overline{n^{-1}} \rightarrow \varphi$.

Let $\Sigma=\left\{\varphi_{i}: i \in I\right\}$ and $n \in \mathbb{N}$. Since $\Sigma \stackrel{s}{=} \varphi$, it follows that for every $\mathcal{L}$ structure $\mathcal{M}$, we have $\sup _{i \in I} \varphi_{i}^{\mathcal{M}} \geq \varphi^{\mathcal{M}}$. Extend the language $\mathcal{L}$ to $\mathcal{L}^{\prime}=\mathcal{L} \cup\{\gamma\}$ where $\gamma$ is a new nullary predicate symbol. Put

$$
\Sigma^{\prime}=\left\{\gamma \Rightarrow \overline{n^{-1}}, \varphi \Rightarrow \gamma\right\} \cup\left\{\gamma \rightarrow \varphi_{i}: i \in I\right\} .
$$

We claim that $\Sigma^{\prime}$ is not satisfiable. Otherwise, let $\mathcal{M}$ be an $\mathcal{L}^{\prime}$-structure which models $\Sigma^{\prime}$. So, $\gamma^{\mathcal{M}}>\widehat{n^{-1}}, \varphi^{\mathcal{M}}>\gamma^{\mathcal{M}}$ and for every $i \in I, \gamma^{\mathcal{M}} \geq \varphi_{i}^{\mathcal{M}}$. Then, $\gamma^{\mathcal{M}} \geq \sup _{i \in I} \varphi_{i}^{\mathcal{M}} \geq \varphi^{\mathcal{M}}$. But, this is a contradiction, since $\varphi^{\mathcal{M}}>\gamma^{\mathcal{M}}$. Therefore, by the compactness theorem, there is a finite subset $\Sigma_{0}^{\prime}$ of $\Sigma$ such that the following theory

$$
\Sigma_{0}^{\prime}=\left\{\gamma \Rightarrow \overline{n^{-1}}, \varphi \Rightarrow \gamma\right\} \cup\left\{\gamma \rightarrow \theta: \theta \in \Sigma_{0}\right\}
$$

is not satisfiable. We claim that $\Sigma_{0} \models s \overline{n^{-1}} \rightarrow \varphi$.

Fix an $\mathcal{L}$-structure $\mathcal{M}$. If $\varphi^{\mathcal{M}} \leq \widehat{n^{-1}}$ then $\left(\overline{n^{-1}} \rightarrow \varphi\right)^{\mathcal{M}}=\hat{0}$ and therefore, $\max _{\theta \in \Sigma_{0}} \theta^{\mathcal{M}} \geq \hat{0}=\left(\overline{n^{-1}} \rightarrow \varphi\right)^{\mathcal{M}}$. So, we may suppose that $\varphi^{\mathcal{M}}>\widehat{n^{-1}}$. We show that $\max _{\theta \in \Sigma_{0}} \theta^{\mathcal{M}} \geq \varphi^{\mathcal{M}}$. If not, then $\varphi^{\mathcal{M}}>\max _{\theta \in \Sigma_{0}} \theta^{\mathcal{M}}$. Choose $\alpha \in \mathbb{I}$ in such a way that

$$
\varphi^{\mathcal{M}}>\alpha>\max _{\theta \in \Sigma_{0}} \theta^{\mathcal{M}},
$$

Then, by interpreting the nullary predicate $\gamma$ by $\alpha$, we may get an $\mathcal{L}^{\prime}$ expansion $\mathcal{N}$ of $\mathcal{M}$ which is a model of $\Sigma^{\prime}$, a contradiction.

Whence, as $\Sigma_{0}$ is finite, by (1) we have $\Sigma_{0} \vdash \overline{n^{-1}} \rightarrow \varphi$. Hence, for any $n \in \mathbb{N}$, $\Sigma \vdash \overline{n^{-1}} \rightarrow \varphi$.

In the following theorem an approximate version of entailment compactness is established.

Theorem 2.24. Let $T$ be an $\mathcal{L}$-theory and $\varphi$ be an $\mathcal{L}$-sentence. $T \models \varphi$ if and only if for every $n \in \mathbb{N}$, there is a finite subset $T_{n}$ of $T$ such that $T_{n} \models \overline{n^{-1}} \rightarrow \varphi$.

Proof. Let $T \models \varphi$. Suppose on the contrary that, there exists $n \in \mathbb{N}$ such that for any finite subset $S$ of $T, S \not \models \overline{n^{-1}} \rightarrow \varphi$. Thus, for any finite subset $S$ of $T$ there is an $\mathcal{L}$-structure $\mathcal{M}$ of $S$ such that $\mathcal{M} \not \models \overline{n^{-1}} \rightarrow \varphi$. So, $\mathcal{M} \models \varphi \rightarrow \overline{n^{-1}}$, i.e., $S \cup\left\{\varphi \rightarrow \overline{n^{-1}}\right\}$ is satisfiable. But, $S$ is an arbitrary finite subset of $T$ and so, $T \cup\left\{\varphi \rightarrow \overline{n^{-1}}\right\}$ is finitely satisfiable. Now, the compactness theorem implies that $T \cup\left\{\varphi \rightarrow \overline{n^{-1}}\right\}$ is satisfiable. But, this contradicts $T \models \varphi$.

Conversely, suppose that for any $n \in \mathbb{N}$ there is a finite subset $T_{n}$ of $T$ such that $T_{n} \models \overline{n^{-1}} \rightarrow \varphi$. We want to show that $T \models \varphi$. Suppose not. Hence, there exists an $\mathcal{L}$-structure $\mathcal{M}$ of $T$ such that $\mathcal{M} \not \models \varphi$. So, there is a natural number $n$ such that $\varphi^{\mathcal{M}} \geq \widehat{n^{-1}}$. This means for any finite subset $S$ of $T, S \not \models \overline{n^{-1}} \rightarrow \varphi$, a contradiction. 
Remark 2.25. Let $u:[0,1]^{2} \rightarrow[0,1]^{2}$ defined by $u(x, y)=(1-x, 1-y)$ and $\mathbb{I}^{*}=[0,1]^{2} \backslash\{(1, r): r<1\}$. One may naturally use the function $u$ to define an $\mathbb{I}^{*}$-interpretation $\mathcal{M}_{u}$ for the language $\mathcal{L}$. Using this function one may translate all semantical issues of $R L G^{*}$, e.g, satisfiability and (strong) entailment to the fuzzy first-order rational Gödel logic. Hence all the results given in this section remain valid for the fuzzy first-order rational Gödel logic.

\section{Ultrametric Logic}

In first-order classical logic the equality relation has the following properties,

(S1) $\forall x(x=x)$,

(S2) $\forall x \forall y(x=y \rightarrow y=x)$,

(S3) $\forall x \forall y \forall z((x=y \wedge y=z) \rightarrow x=z)$,

(E1) for all function symbol $f, \forall \bar{x} \forall \bar{y}((\bar{x}=\bar{y}) \rightarrow(f(\bar{x})=f(\bar{y})))$,

(E2) for all predicate symbol $P, \forall \bar{x} \forall \bar{y}((\bar{x}=\bar{y}) \rightarrow(P(\bar{x}) \leftrightarrow P(\bar{y})))$.

While (S1-S3) are called the similarity axioms, (E1,E2) are named the extensionality axioms. The next lemma shows the meaning of the above axioms in $R G L^{*}$.

Lemma 3.1. Let $\mathcal{M}$ be an $\mathcal{L}$-structure and suppose " $d$ " is a distinguished binary predicate symbol in $\mathcal{L}$.

1) If $d^{\mathcal{M}}$ satisfies the similarity axioms then $d^{\mathcal{M}}$ defines an extended pseudoultrametric on the universe of $\mathcal{M}$, i.e., $d^{\mathcal{M}}: M^{2} \rightarrow \mathbb{I}$ satisfies the following properties, for all $a, b, c, \in M$ :

$$
\begin{gathered}
d^{\mathcal{M}}(a, a)=\hat{0}, \\
d^{\mathcal{M}}(a, b)=d^{\mathcal{M}}(b, a), \\
d^{\mathcal{M}}(a, b) \leq \max \left\{d^{\mathcal{M}}(a, c), d^{\mathcal{M}}(b, c)\right\} .
\end{gathered}
$$

2) Moreover, if $\mathcal{M}$ satisfies the extensionality axioms then the interpretation of any function symbol $f$ and any predicate symbol $P$ is 1-Lipschitz, respectively, i.e., for all $\bar{a}, \bar{b} \in M^{n}$,

$$
\begin{gathered}
d^{\mathcal{M}}\left(f^{\mathcal{M}}(\bar{a}), f^{\mathcal{M}}(\bar{b})\right) \leq d^{\mathcal{M}}(\bar{a}, \bar{b}), \\
d_{\text {max }}\left(P^{\mathcal{M}}(\bar{a}), P^{\mathcal{M}}(\bar{b})\right) \leq d^{\mathcal{M}}(\bar{a}, \bar{b}) .
\end{gathered}
$$

Remark 3.2. For a given extended pseudo-ultrametric space $\left(M, d: M^{2} \rightarrow \mathbb{I}\right)$, one may define an extended pseudo-ultrametric $d_{n}(\bar{a}, \bar{b})$ on the set of $n$-tuples of $M^{n}$ as

$$
d_{n}(\bar{a}, \bar{b})=\max \left\{d^{\mathcal{M}}\left(a_{i}, b_{i}\right): 1 \leq i \leq n\right\} .
$$

We may omit the subscript $n$ if there is no danger of ambiguity. 
We are interested in obtaining an extended ultrametric instead of an extended pseudo-ultrametric. To this end, we need a weaker translation of the extensionality axioms.

Following continuous first-order logic, we replace $(\mathrm{E} 1, \mathrm{E} 2)$ by somewhat weaker axioms, ensuring that the interpretation of all function and predicate symbols are uniformly continuous, i.e., for any $n \in \mathbb{N}$, there exist $m_{P}, m_{f} \in \mathbb{N}$ such that for all $\bar{a}, \bar{b} \in M$,
1) if $d^{\mathcal{M}}(\bar{a}, \bar{b})<\widehat{m_{f}^{-1}}$ then $d^{\mathcal{M}}\left(f^{\mathcal{M}}(\bar{a}), f^{\mathcal{M}}(\bar{b})\right) \leq \widehat{n^{-1}}$,
2) if $d^{\mathcal{M}}(\bar{a}, \bar{b})<\widehat{m_{P}^{-1}}$ then $d_{\max }\left(P^{\mathcal{M}}(\bar{a}), P^{\mathcal{M}}(\bar{b})\right) \leq \widehat{n^{-1}}$.

Definition 3.3. A first-order language $\mathcal{L}$ with a distinguished binary relation " $d$ " together with a set of modulus of uniform continuity functions $\left\{\delta_{e}: \mathbb{N} \rightarrow \mathbb{N}\right.$ : $e$ is either a function or predicate symbol $\}$ is called a continuous ultrametric language.

From now on, assume $\mathcal{L}$ is a fixed continuous ultrametric language. Now, the notion of $\mathcal{L}$-structures is modified accordingly.

Definition 3.4. A continuous ultrametric $\mathcal{L}$-pre-structure or simply an ultrametric $\mathcal{L}$-pre-structure is an $\mathcal{L}$-structure $\mathcal{M}$ with the following properties:

1. $d^{\mathcal{M}}: M^{2} \rightarrow \mathbb{I}$ is an extended pseudo-ultrametric,

2. for every $n$-ary function symbol " $f ", f^{\mathcal{M}}:\left(M^{n}, d^{\mathcal{M}}\right) \rightarrow\left(M, d^{\mathcal{M}}\right)$ satisfies the following condition for any $n \in \mathbb{N}$ and all $\bar{a}, \bar{b} \in M$,

$$
\text { if } d^{\mathcal{M}}(\bar{a}, \bar{b})<\left(\widehat{\left.\delta_{f}(n)\right)^{-1}} \text { then } d^{\mathcal{M}}(f(\bar{a}), f(\bar{b}))<\widehat{n^{-1}},\right.
$$

3. for every $n$-ary predicate symbol " $P$ ", $P^{\mathcal{M}}:\left(M^{n}, d^{\mathcal{M}}\right) \rightarrow\left(\mathbb{I}, d_{\max }\right)$ satisfies the following condition for any $n \in \mathbb{N}$ and all $\bar{a}, \bar{b} \in M$,

$$
\text { if } d^{\mathcal{M}}(\bar{a}, \bar{b})<\left(\widehat{\left.\delta_{P}(n)\right)^{-1}} \text { then } d_{\max }\left(P^{\mathcal{M}}(\bar{a}), P^{\mathcal{M}}(\bar{b})\right)<\widehat{n^{-1}}\right. \text {. }
$$

Remark 3.5. An extended pseudo-ultrametric $d: M^{2} \rightarrow \mathbb{I}$ on the set $M$ is called an extended ultrametric, if $d(a, b)=\hat{0}$ implies that $a=b$ for any $a, b \in M$.

Following continuous first-order logic [4, the next definition is established.

Definition 3.6. $A$ continuous ultrametric $\mathcal{L}$-structure or simply an ultrametric $\mathcal{L}$-structure is a ultrametric $\mathcal{L}$-pre-structure $\mathcal{M}$, where $d^{\mathcal{M}}: M^{2} \rightarrow \mathbb{I}$ is an extended ultrametric.

Lemma 3.7. Suppose $\mathcal{M}$ is an ultrametric $\mathcal{L}$-pre-structure. Then, there exist an ultrametric $\mathcal{L}$-structure $\mathcal{M}_{0}$ and a function $f: M \rightarrow M_{0}$ such that for any $\mathcal{L}$-formula $\varphi(\bar{x})$ and $\bar{a} \in M^{n}$,

$$
\mathcal{M} \models \varphi(\bar{a}) \text { iff } \mathcal{M}_{0} \models \varphi(f(\bar{a})) .
$$

Proof. Define the equivalence relation $\sim$ as

$$
a \sim b \text { iff } d^{\mathcal{M}}(a, b)=\hat{0} .
$$


Denote the equivalence class of $a \in M$ by $[a]_{\sim}$. Now, let $M_{0}$ be the set of the equivalence classes of $\sim$ in $M$. Define

$$
d^{\mathcal{M}_{0}}\left([a]_{\sim},[b]_{\sim}\right)=d^{\mathcal{M}}(a, b) .
$$

Then, $\left(M_{0}, d^{\mathcal{M}_{0}}\right)$ is an extended ultrametric space. Furthermore, for any predicate and function symbols $P$ and $f$ set

$$
\begin{aligned}
P^{\mathcal{M}}\left(\left[a_{1}\right]_{\sim}, \ldots,\left[a_{n_{P}}\right]_{\sim}\right) & =P^{\mathcal{M}}\left(a_{1}, \ldots, a_{n_{P}}\right), \\
f^{\mathcal{M}}\left(\left[a_{1}\right]_{\sim}, \ldots,\left[a_{n_{f}}\right]_{\sim}\right) & =f^{\mathcal{M}}\left(a_{1}, \ldots, a_{n_{f}}\right) .
\end{aligned}
$$

The uniform continuity of the interpretation of predicate and function symbols in $\mathcal{M}$ implies that the above definitions are well-defined. So, if predicate and function symbols satisfy the uniform continuity axioms then we get an $\mathcal{L}$-structure $\mathcal{M}_{0}$.

Define $f: M \rightarrow M_{0}$ as $f(a)=[a]_{\sim}$. Obviously, $\varphi^{\mathcal{M}_{0}}\left(\left[a_{1}\right]_{\sim}, \ldots,\left[a_{n}\right]_{\sim}\right)=$ $\varphi^{\mathcal{M}}\left(a_{1}, \ldots, a_{n}\right)$ for any $\mathcal{L}$-formula $\varphi(\bar{x})$ and $\bar{a} \in M^{n}$. Whence, $f$ is the desirable function.

The axiom system of ultrametric logic consists of the axioms of $R G L^{*}$ together with the following axioms:

S1. $\forall x(d(x, x))$,

S2. $\forall x \forall y(d(x, y) \rightarrow d(y, x))$,

S3. $\forall x \forall y \forall z((d(x, y) \wedge d(y, z)) \rightarrow d(x, z))$,

for any function symbol $f$, and any predicate symbol $P$ and any natural number $n$,

WE1. $\forall \bar{x} \forall \bar{y}\left(\left(d(\bar{x}, \bar{y}) \rightarrow \overline{\delta_{f}(n)}\right) \vee\left(\overline{n^{-1}} \rightarrow d(f(\bar{x}), f(\bar{y}))\right)\right)$,

WE2. $\forall \bar{x} \forall \bar{y}\left(\left(d(\bar{x}, \bar{y}) \rightarrow \overline{\delta_{P}(n)}\right) \vee\left(\overline{n^{-1}} \rightarrow(p(\bar{x}) \leftrightarrow p(\bar{y}))\right)\right)$.

The inference rules are the same as $R G L^{*}$. The ultrametric logic is denoted by UML.

Corollary 3.8. Any strongly consistent (resp. finitely satisfiable) theory is satisfiable in $U M L$.

\section{Some Model Theory for Ultrametric Logic}

In this section, some model theory for UML is developed. Using the machinery developed here, the Robinson consistency theorem (Theorem 4.15) is proved. To simplify the notation, by an $\mathcal{L}$-structure we mean an ultrametric $\mathcal{L}$-structure.

Definition 4.1. Let $\mathcal{M}$ and $\mathcal{N}$ be two $\mathcal{L}$-structures.

1. $\mathcal{M}$ and $\mathcal{N}$ are elementary equivalent, denoted by $\mathcal{M} \equiv \mathcal{N}$, if $\varphi^{\mathcal{M}}=\varphi^{\mathcal{N}}$ for any $\varphi \in \operatorname{Sent}(\mathcal{L})$.

2. An embedding is a function $j: M \rightarrow N$ with the following properties. For any function symbol $f$ and predicate symbol $P$, 
a) $j\left(f^{\mathcal{M}}\left(a_{1}, \ldots, a_{n_{f}}\right)\right)=f^{\mathcal{N}}\left(j\left(a_{1}\right), \ldots, j\left(a_{n_{f}}\right)\right), f \in \mathcal{L}$ and $\bar{a} \in M^{n_{f}}$,

b) $P^{\mathcal{M}}\left(a_{1}, \ldots, a_{n_{P}}\right)=P^{\mathcal{N}}\left(j\left(a_{1}\right), \ldots, j\left(a_{n_{P}}\right)\right), P \in \mathcal{L}$ and $\bar{a} \in M^{n_{P}}$.

It is easy to see that for every quantifier-free $\mathcal{L}$-formula $\varphi(\bar{x})$ and any element $\bar{a} \in M^{n}$,

$(*) \quad \varphi^{\mathcal{M}}\left(a_{1}, \ldots, a_{n}\right)=\varphi^{\mathcal{N}}\left(j\left(a_{1}\right), \ldots, j\left(a_{n}\right)\right)$.

Moreover, an embedding is an isometry, i.e., $d^{\mathcal{M}}(a, b)=d^{\mathcal{N}}(j(a), j(b))$ for any $a, b, \in M$. Therefore, $j$ is injective.

3. The embedding $j$ is elementary, if (*) holds for any $\mathcal{L}$-formula $\varphi(\bar{x})$. In this case, $j$ is denoted by $j: \mathcal{M} \hookrightarrow \mathcal{N}$.

4. An isomorphism is a surjective elementary embedding.

Remark 4.2. The notion of substructure (resp. elementary substructure) is a special case of the above definition, where $M \subseteq N$ and the inclusion map is embedding (resp. elementary embedding). In this case, we write $\mathcal{M} \subseteq \mathcal{N}$ (resp. $\mathcal{M} \preceq \mathcal{N})$.

Remark 4.3. As in the first-order logic, $T h_{\mathcal{L}}(\mathcal{M})$ is the full theory of $\mathcal{L}$ structure $\mathcal{M}$, that $T h_{\mathcal{L}}(\mathcal{M})=\{\varphi \in \operatorname{Sent}(\mathcal{L}, \mathcal{A}): \mathcal{M} \models \varphi\}$.

While in first-order logic or even in continuous first-order logic the notion of embedding (resp. elementary embedding) can be captured by means of the notion of diagram (resp. elementary diagram), the lack of such feature for ultrametric logic leads to the following definitions.

Definition 4.4. Let $\mathcal{M}$ be an $\mathcal{L}$-structure, $\varphi(\bar{x}) \in \operatorname{Form}(\mathcal{L})$ and $\bar{a} \in M$. The truth degree of $\varphi^{\mathcal{M}}(\bar{a})$, st $t_{\mathcal{M}}(\varphi(\bar{a}))$, is defined by

$$
\inf \left\{r \in[0,1]_{\mathbb{Q}}: \mathcal{M} \models \bar{r} \rightarrow \varphi(\bar{a})\right\} \in[0,1] .
$$

Definition 4.5. Let $\mathcal{M}$ and $\mathcal{N}$ be two $\mathcal{L}$-structures.

1. $\mathcal{M}$ and $\mathcal{N}$ are weakly elementary equivalent, $\mathcal{M} \stackrel{w}{=} \mathcal{N}$, if $\operatorname{st}_{\mathcal{M}}(\varphi)=s t_{\mathcal{N}}(\varphi)$ for any $\mathcal{L}$-sentence $\varphi$. Observe that if $\mathcal{M} \stackrel{w}{=} \mathcal{N}$ then for every two $\mathcal{L}$ sentences $\varphi$ and $\psi$,

$$
\varphi^{\mathcal{M}} \leq \psi^{\mathcal{M}} \text { iff } \varphi^{\mathcal{N}} \leq \psi^{\mathcal{N}}
$$

2. A function $j: M \rightarrow N$ is called a weak elementary $\mathcal{L}$-embedding, denoted by $j: \mathcal{M} \stackrel{w}{\hookrightarrow}_{\mathcal{L}} \mathcal{N}$, if it has the following properties,

a) $j\left(f^{\mathcal{M}}\left(a_{1}, \ldots, a_{n_{f}}\right)\right)=f^{\mathcal{N}}\left(j\left(a_{1}\right), \ldots, j\left(a_{n_{f}}\right)\right), f \in \mathcal{L}$ and $\bar{a} \in M^{n_{f}}$,

b) $\operatorname{st}_{\mathcal{M}}\left(\varphi\left(a_{1}, \ldots, a_{n}\right)\right)=s t_{\mathcal{N}}\left(\varphi\left(j\left(a_{1}\right), \ldots, j\left(a_{n}\right)\right)\right), \varphi(\bar{x}) \in \operatorname{Form}(\mathcal{L})$ and $\bar{a} \in M^{n}$.

3. Let $\mathcal{L}(M)=\mathcal{L} \cup\left\{c_{m}: m \in M\right\}$, where $\left\{c_{m}\right\}_{m \in M}$ are some new constant symbols. One can naturally interpret any $c_{m}$ inside the $\mathcal{L}$-structure $\mathcal{M}$ by m. So, any $\mathcal{L}$-structure $\mathcal{M}$ can be viewed as an $\mathcal{L}(M)$-structure. Now, the weak elementary diagram of $\mathcal{M}$ is 


$$
\operatorname{wediag}_{\mathcal{L}}(\mathcal{M})=T h_{\mathcal{L}(M)}(\mathcal{M}) .
$$

If there is no danger of confusion we may drop the subscript $\mathcal{L}$ and simply write $j: \mathcal{M} \stackrel{w}{\leftrightarrow} \mathcal{N}$ and $\operatorname{wediag}(\mathcal{M})$.

Remark 4.6. Note that if $j: \mathcal{M} \stackrel{w}{\rightarrow} \mathcal{N}$, then whenever $j(a)=j(b)$ for $a, b \in M$, we have $d^{\mathcal{N}}(j(a), j(b))=\hat{0}$. Hence, $d^{\mathcal{M}}(a, b)=\hat{0}$ which implies $a=b$. So, $j$ is an injective function.

The following lemma justifies why we call $T h_{\mathcal{L}(M)}(\mathcal{M})$ a weak elementary diagram, and why we need the additional concepts that are defined in Definition 4.5

Lemma 4.7. Let $\mathcal{M}$ be an $\mathcal{L}$-structure and $\mathcal{N}$ be an $\mathcal{L}(M)$-structure such that $\mathcal{N} \models$ wediag $(\mathcal{M})$. Then, there is a weak elementary $\mathcal{L}$-embedding $j: \mathcal{M} \stackrel{w}{\rightarrow} \mathcal{N}$.

Proof. Clear.

Lemma 4.8. Let $\mathcal{M}, \mathcal{N}$ and $\mathcal{B}$ be three countable $\mathcal{L}$-structures and $i: \mathcal{M} \stackrel{w}{\rightarrow} \mathcal{B}$ and $j: \mathcal{N} \stackrel{w}{\hookrightarrow} \mathcal{B}$ be weak elementary embeddings. Then, there is an $\mathcal{L}$-structure $\mathcal{B}^{\prime}$ with the same universe as $\mathcal{B}$ such that $\mathcal{M} \stackrel{w}{\hookrightarrow} \mathcal{B}^{\prime}$ and $\mathcal{N} \hookrightarrow \mathcal{B}^{\prime}$.

Proof. Since $j$ is a weak elementary embedding, the following two subsets of $\mathbb{I}$

$$
\begin{gathered}
A=\left\{\varphi^{\mathcal{N}}\left(a_{1}, \ldots, a_{n}\right): \varphi\left(x_{1}, \ldots, x_{n}\right) \in F \operatorname{Form}(\mathcal{L}), a_{1}, \ldots, a_{n} \in N\right\}, \\
B=\left\{\varphi^{\mathcal{B}}\left(j\left(a_{1}\right), \ldots, j\left(a_{n}\right)\right): \varphi\left(x_{1}, \ldots, x_{n}\right) \in \operatorname{Form}(\mathcal{L}), a_{1}, \ldots, a_{n} \in N\right\}
\end{gathered}
$$

satisfy the following properties:

1) $\varphi^{\mathcal{N}}\left(a_{1}, \ldots, a_{n}\right)<\psi^{\mathcal{N}}\left(b_{1}, \ldots, b_{m}\right)$ iff $\varphi^{\mathcal{B}}\left(j\left(a_{1}\right), \ldots, j\left(a_{n}\right)\right)<\psi^{\mathcal{B}}\left(j\left(b_{1}\right), \ldots, j\left(b_{m}\right)\right)$,

2) $\varphi^{\mathcal{N}}\left(a_{1}, \ldots, a_{n}\right)=\psi^{\mathcal{N}}\left(b_{1}, \ldots, b_{m}\right)$ iff $\varphi^{\mathcal{B}}\left(j\left(a_{1}\right), \ldots, j\left(a_{n}\right)\right)=\psi^{\mathcal{B}}\left(j\left(b_{1}\right), \ldots, j\left(b_{m}\right)\right)$.

By the same idea used in Theorem 2.19, one may find a continuous order preserving function $h: \mathbb{I} \rightarrow \mathbb{I}$ such that

1) $h(\hat{r})=\hat{r}$

2) $h\left(\varphi^{\mathcal{B}}\left(j\left(a_{1}\right), \ldots, j\left(a_{n}\right)\right)\right)=\varphi^{\mathcal{N}}\left(a_{1}, \ldots, a_{n}\right)$.

Now, define an $\mathcal{L}$-structure $\mathcal{B}^{\prime}$ in such a way that the interpretations of constant and function symbols are the same as $\mathcal{B}$ and for any predicate $P$, we have $P^{\mathcal{B}^{\prime}}(\bar{b})=h\left(P^{\mathcal{B}}(\bar{b})\right)$. A simple induction shows that for any formula $\varphi\left(x_{1}, \ldots, x_{n}\right)$ and $b_{1}, \ldots, b_{n} \in B, \varphi^{\mathcal{B}^{\prime}}(\bar{b})=h\left(\varphi^{\mathcal{B}}(\bar{b})\right)$. Moreover, the function $j: \mathcal{N} \rightarrow \mathcal{B}^{\prime}$ is an elementary embedding, while the function $i: \mathcal{M} \rightarrow \mathcal{B}^{\prime}$ is a weak elementary embedding.

The above proof highlights a special but important instant of the notion of weak elementary equivalence.

Definition 4.9. Let $\mathcal{M}$ and $\mathcal{M}^{\prime}$ be two $\mathcal{L}$-structures with the same universe $M$. Suppose $h: \mathbb{I} \rightarrow \mathbb{I}$ is an order preserving function. We say that $\mathcal{M}$ and $\mathcal{M}^{\prime}$ are $h$-equivalent if $\varphi^{\mathcal{M}^{\prime}}(\bar{a})=h\left(\varphi^{\mathcal{M}}(\bar{a})\right)$. In this case, we write $\mathcal{M} \equiv_{h} \mathcal{M}^{\prime}$. 
Theorem 4.10. (Amalgamation property) Let $\mathcal{B}, \mathcal{D}$ and $\mathcal{E}$ be three countable $\mathcal{L}$-structures. Suppose also, $j: \mathcal{E} \stackrel{w}{\hookrightarrow} \mathcal{B}$ and $k: \mathcal{E} \stackrel{w}{\hookrightarrow} \mathcal{D}$ are weak elementary embeddings. Then, there are an $\mathcal{L}$-structure $\mathcal{G}$ and embeddings $j_{1}: \mathcal{B} \stackrel{w}{\longrightarrow} \mathcal{G}$ and $k_{1}: \mathcal{D} \hookrightarrow \mathcal{G}$ such that $j_{1} \circ j=k_{1} \circ k$.

Proof. Let $\mathcal{L}_{B}=\mathcal{L}(E) \cup\left\{c_{b}: b \in B \backslash j(E)\right\}, \mathcal{L}_{D}=\mathcal{L}(E) \cup\left\{c_{d}: d \in D \backslash j(E)\right\}$ and $\mathcal{L}^{\prime}=\mathcal{L}_{B} \cup \mathcal{L}_{D}$. Without loss of generality, we may assume that $\mathcal{L}_{B} \cap \mathcal{L}_{D}=\mathcal{L}(E)$. One can naturally interpret the new constants $c_{e}$ and $c_{b}$ for $e \in E$ and $b \in B$ inside the $\mathcal{L}$-structure $\mathcal{B}$ by $j(e)$ and $b$, respectively. Also, $\mathcal{B}$ can be considered as an $\mathcal{L}_{B}$-structure. We want to show that wediag $(\mathcal{B}) \cup$ wediag $(\mathcal{D})$ is a satisfiable $\mathcal{L}^{\prime}$-theory.

For a given $\varphi\left(c_{e_{1}}, \ldots, c_{e_{n}}, c_{b_{1}}, \ldots, c_{b_{m}}\right) \in \operatorname{wediag}(\mathcal{B})$, we have $\mathcal{B} \models \exists \bar{x} \varphi\left(c_{e_{1}}, \ldots, c_{e_{n}}, \bar{x}\right)$. So, $\mathcal{B} \models \exists \bar{x} \varphi\left(j\left(e_{1}\right), \ldots, j\left(e_{n}\right), \bar{x}\right)$. Moreover, since $j: \mathcal{E} \stackrel{w}{\hookrightarrow} \mathcal{B}$ and $k: \mathcal{E} \stackrel{w}{\hookrightarrow} \mathcal{D}$, it follows that $\mathcal{E} \models \exists \bar{x} \varphi\left(e_{1}, \ldots, e_{n}, \bar{x}\right)$ and therefore, $\mathcal{D} \models \exists \bar{x} \varphi\left(k\left(e_{1}\right), \ldots, k\left(e_{n}\right), \bar{x}\right)$, i.e., $\inf _{\bar{d} \in D} \varphi^{\mathcal{D}}\left(k\left(e_{1}\right), \ldots, \bar{d}\right)=\hat{0}$. Hence, for any rational number $0<r \leq 1$, there exists $\bar{d}_{r} \in D$ such that $\varphi^{\mathcal{D}}\left(k\left(e_{1}\right), \ldots, \bar{d}_{r}\right) \leq \hat{r}$. Thus, the following set

$$
\operatorname{wediag}(\mathcal{D}) \cup\left\{\bar{r} \rightarrow \varphi\left(c_{e_{1}}, \ldots, c_{e_{n}}, c_{b_{1}}, \ldots, c_{b_{m}}\right): 0<r \leq 1\right\}
$$

is finitely satisfiable. Therefore, by the Lemma 2.13

$$
\operatorname{wediag}(\mathcal{D}) \cup\left\{\varphi\left(c_{e_{1}}, \ldots, c_{e_{n}}, c_{b_{1}}, \ldots, c_{b_{m}}\right)\right\}
$$

is strongly consistent. This leads us to show that $\operatorname{wediag}(\mathcal{B}) \cup \operatorname{wediag}(\mathcal{D})$ is satisfiable.

Now, any model $\mathcal{G} \models \operatorname{wediag}(\mathcal{B}) \cup \operatorname{wediag}(\mathcal{D})$ gives two weak elementary embeddings $j_{1}: \mathcal{B} \stackrel{w}{\hookrightarrow} \mathcal{G}$ and $k_{1}: \mathcal{D} \stackrel{w}{\hookrightarrow} \mathcal{G}$ such that $j_{1} \circ j=k_{1} \circ k$. Furthermore, by Lemma 4.8, $k_{1}$ can be assumed to be an elementary embedding.

Below, the notion of direct limit of a family of sets and functions is defined and then, using this concept, the notion of pseudo-direct limit of a weak elementary chain of $\mathcal{L}$-structures is introduced.

Definition 4.11. Let $\left(M_{i}\right)_{i \in \mathbb{N}}$ be a family of pairwise disjoint sets. Suppose also $\left(f_{i, j}: M_{i} \rightarrow M_{j}\right)_{i \leq j \in \mathbb{N}}$ is a family of functions. Call $\mathcal{F}=\left\{\left(M_{i}\right)_{i \in \mathbb{N}},\left(f_{i, j}\right)_{i \leq j \in \mathbb{N}}\right\}$ $a$ direct system, if the following properties hold:

$$
\begin{aligned}
& f_{i, i}=i d_{M_{i}} \text { for any } i \in \mathbb{N}, \\
& f_{i, j}=f_{k, j} \circ f_{i, k} \text { for any } i \leq k \leq j \in \mathbb{N} .
\end{aligned}
$$

For any direct system $\mathcal{F}=\left\{\left(M_{i}\right)_{i \in \mathbb{N}},\left(f_{i, j}\right)_{i \leq j \in \mathbb{N}}\right\}$, define an equivalence relation $\equiv_{\mathcal{F}}$ on $M_{\infty}=\bigcup_{i \in \mathbb{N}} M_{i}$ as follows. For any $a \in M_{i}$ and $b \in M_{j}$,

$$
a \equiv_{\mathcal{F}} b \text { iff there exists } k \geq i, j \text { such that } f_{i, k}(a)=f_{j, k}(b) .
$$

The equivalence classes of $\equiv_{\mathcal{F}}$ is denoted by $[a]_{\mathcal{F}}$. The set of all equivalence classes of $\equiv_{\mathcal{F}}$ is also denoted by $\lim _{\mathcal{F}} M_{i}$.

It is customary to call $\lim _{\mathcal{F}} M_{i}$ the direct limit of the direct system $\mathcal{F}$.

Definition 4.12. Suppose that $\left(\mathcal{M}_{i}\right)_{i \in \mathbb{N}}$ is a family of $\mathcal{L}$-structures and $\left(f_{i, j}\right.$ : $\left.\mathcal{M}_{i} \stackrel{w}{\hookrightarrow} \mathcal{M}_{j}\right)_{i \leq j \in \mathbb{N}}$ is a family of weak elementary embeddings. We call $\mathfrak{F}=$ $\left\{\mathcal{M}_{i}, f_{i, j}\right\}$ a weak direct system of $\mathcal{L}$-structures if $\mathcal{F}=\left\{\left(M_{i}\right)_{i \in \mathbb{N}},\left(f_{i, j}\right)_{i \leq j \in \mathbb{N}}\right\}$ is a direct system. 
Lemma 4.13. (Union of chain) Let $\mathfrak{F}=\left\{\mathcal{M}_{i}, f_{i, j}\right\}$ be a weak direct system of $\mathcal{L}$-structures. There exist an $\mathcal{L}$-structure $\mathcal{M}_{\infty}$ whose underlying universe is $\lim _{\mathcal{F}} M_{i}$ and a family $\left\{h_{i}: \mathcal{M}_{i} \stackrel{w}{\longrightarrow} \mathcal{M}_{\infty}\right\}_{i \in \mathbb{N}}$ of weak elementary embeddings such that $h_{j} \circ f_{i, j}=h_{i}$ for any $i \leq j \in \mathbb{N}$.

Proof. For any $i \in \mathbb{N}$, let $C_{i}=\left\{c_{m}: m \in M_{i}\right\}$ be a set of new constant symbols and suppose further, that $\left(C_{i}\right)_{i \in \mathbb{N}}$ are pairwise disjoint. Let $\mathcal{L}_{n}=\mathcal{L} \cup \bigcup_{i=1}^{n} C_{i}$ and $\mathcal{L}_{\infty}=\mathcal{L} \cup \bigcup_{i=1}^{\infty} C_{i}$. Also, for any $i \leq j \in \mathbb{N}$, the function $\widetilde{f}_{i, j}: C_{i} \rightarrow C_{j}$ is defined by $\widetilde{f}_{i, j}\left(c_{m}\right)=c_{f_{i, j}(m)}$.

For every $i \in \mathbb{N}$, set

$$
\begin{gathered}
\Gamma_{i}=\left\{\varphi\left(c_{m_{1}}, \ldots, c_{m_{n}}\right): \varphi\left(m_{1}, \ldots, m_{n}\right) \in \operatorname{wediag}\left(\mathcal{M}_{i}\right)\right\} \\
\Delta_{i}=\left\{d\left(c_{m}, \widetilde{f}_{i, j}\left(c_{m}\right)\right): i \leq j \in \mathbb{N}, c_{m} \in C_{i}\right\} .
\end{gathered}
$$

Also, for every $\mathcal{L}_{\infty}$-formula $\varphi(x, \bar{c})$, take $n_{\varphi}$ to be the least natural number such that $\varphi(x, \bar{c})$ is an $\mathcal{L}_{n_{\varphi}}$-formula and put

$$
\widetilde{\varphi}(x, \bar{c})=\varphi\left(x, \widetilde{f}_{i_{1}, n_{\varphi}}\left(c_{m_{i_{1}}}\right), \ldots, c_{m_{n_{\varphi}}}\right),
$$

where $\bar{c}=\left(c_{m_{i_{1}}}, \ldots, c_{m_{n_{\varphi}}}\right)$ and $i_{1} \leq \ldots \leq n_{\varphi}$. For any $i \in \mathbb{N}$, let

$$
S_{i}=\left\{\forall x \varphi(x, \bar{c}): \varphi(x, \bar{c}) \in \operatorname{Form}\left(\mathcal{L}_{i}\right) \text { and } \forall x \widetilde{\varphi}(x, \bar{c}) \in \Gamma_{n_{\varphi}}\right\} .
$$

Note that if $\forall x \varphi(x, \bar{c}) \in S_{i}$ then $n_{\varphi} \leq i$. In the sequel, we show that

$$
\Sigma=\bigcup_{i=1}^{\infty} \Gamma_{i} \cup \bigcup_{i=1}^{\infty} \Delta_{i} \cup \bigcup_{i=1}^{\infty} S_{i}
$$

is a satisfiable Henkin $\mathcal{L}_{\infty}$-theory and has a model $\mathcal{M}$ with the universe $M=$ $\lim _{\widetilde{\mathcal{F}}} C_{i}$, where $\widetilde{\mathcal{F}}=\left\{\left(C_{i}\right)_{i \in \mathbb{N}},\left(\widetilde{f}_{i, j}\right)_{i \leq j \in \mathbb{N}}\right\}$.

To show that $\Sigma$ is satisfiable, for any $n \in \mathbb{N}$, consider the $\mathcal{L}_{n}$-theory

$$
\Sigma_{n}=\bigcup_{i=1}^{n} \Gamma_{i} \cup \bigcup_{i=1}^{n} \Delta_{i} \cup \bigcup_{i=1}^{n} S_{i} .
$$

Let $\widehat{\mathcal{M}}_{n}$ be the expansion of the $\mathcal{L}$-structure $\mathcal{M}_{n}$ to an $\mathcal{L}_{n}$-structure such that $c_{m}^{\widehat{\mathcal{M}}_{n}}=f_{i, n}(m)$ for any $c_{m} \in C_{i}, i \leq n$. We claim that $\widehat{\mathcal{M}}_{n} \models d\left(c_{m}, \widetilde{f}_{i, n}\left(c_{m}\right)\right)$. To see this let $m^{\prime}=f_{i, n}(m)$. Since for any $c_{m} \in C_{i}, f_{i, n}(m) \in C_{n}$, we have

$$
\left(\widetilde{f}_{i, n}\left(c_{m}\right)\right)^{\widehat{\mathcal{M}}_{n}}=c_{m^{\prime}}^{\widehat{\mathcal{M}}_{n}}=f_{n, n}\left(m^{\prime}\right)=f_{n, n}\left(f_{i, n}(m)\right)=f_{i, n}(m) .
$$

So, $\widehat{\mathcal{M}}_{n} \models \Delta_{i}$ for any $i \leq n$.

Furthermore, for any $n \geq n_{\varphi}$ whenever $\forall x \varphi(x, \bar{c}) \in \bigcup_{i=1}^{n} S_{i}, \forall x \widetilde{\varphi}(x, \bar{c}) \in \Gamma_{n_{\varphi}}$. Hence, $\mathcal{M}_{n_{\varphi}} \models \forall x \widetilde{\varphi}(x, \bar{c})$. This implies $\widehat{\mathcal{M}}_{n} \models \forall x \varphi(x, \bar{c})$ and $\widehat{\mathcal{M}}_{n} \models \Sigma_{n}$. So, $\Sigma_{n}$ is satisfiable and by the compactness theorem, $\Sigma$ is satisfiable.

Secondly, to verify that $\Sigma$ is Henkin, let $\Sigma \nvdash \forall x \varphi(x, \bar{c})$. In particular, $\forall x \varphi(x, \bar{c}) \notin$ $\Sigma$. So, $\forall x \widetilde{\varphi}(x, \bar{c}) \notin \Gamma_{n_{\varphi}}$ and therefore, $(\forall x \widetilde{\varphi}(x, \bar{c}))^{\mathcal{M}_{n_{\varphi}}}>\hat{0}$. This means that there is a rational number $r>0$ such that $(\forall x \widetilde{\varphi}(x, \bar{c}))^{\mathcal{M}_{n_{\varphi}}}>\hat{r}$. Thus, there exists $d \in M_{n_{\varphi}}$ such that $\left(\widetilde{\varphi}\left(c_{d}, \bar{c}\right)\right)^{\mathcal{M}_{n_{\varphi}}} \geq \hat{r}$. So, $\left(\widetilde{\varphi}\left(c_{d}, \bar{c}\right) \rightarrow \bar{r}\right)^{\mathcal{M}_{n_{\varphi}}}=\hat{0}$, that is

$$
\left(\varphi\left(c_{d}, \bar{c}\right) \rightarrow \bar{r}\right)^{\widehat{\mathcal{M}}_{n \varphi}}=\hat{0} .
$$


Hence, $\Sigma \nvdash \varphi\left(c_{d}, \bar{c}\right)$. If not, then for some $n \geq n_{\varphi}, \Sigma_{n} \vdash \varphi\left(c_{d}, \bar{c}\right)$. Thus, by soundness $\Sigma_{n} \models \varphi\left(c_{d}, \bar{c}\right)$. Now, since $\widehat{\mathcal{M}}_{n} \models \Sigma_{n}$ it follows that $\varphi^{\widehat{\mathcal{M}}_{n}}\left(c_{d}, \bar{c}\right)=\hat{0}$. But, this contradicts with (7).

Take an $\mathcal{L}_{\infty}$-theory $\Sigma^{\prime}$ to be a maximally linear complete extension of $\Sigma$. This extension remains Henkin. If $\Sigma^{\prime} \nvdash \forall x \varphi\left(x, c_{m_{1}}, \ldots, c_{m_{n}}\right)$ then $\Sigma \nvdash \forall x \varphi\left(x, c_{m_{1}}, \ldots, c_{m_{n}}\right)$. Now, as $\Sigma$ is Henkin, by a similar argument used in the above paragraph there exist a constant $c$ and a rational number $r>0$ such that $\varphi\left(c, c_{m_{1}}, \ldots, c_{m_{n}}\right) \rightarrow$ $\bar{r} \in \Sigma$. Thus, $\Sigma^{\prime} \vdash \varphi\left(c, c_{m_{1}}, \ldots, c_{m_{n}}\right) \rightarrow \bar{r}$, that is $\Sigma^{\prime} \nvdash \varphi\left(c, c_{m_{1}}, \ldots, c_{m_{n}}\right)$.

The method used in the proof of the completeness theorem implies that there is an $\mathcal{L}_{\infty}$-structure $\mathcal{M}$ whose universe is the interpretation of the set of constant symbols $\cup_{i \in \mathbb{N}} C_{i}$. One can easily see that $M=\lim _{\mathcal{F}} M_{i}$. And, moreover, for any $i \in \mathbb{N}$, the function $h_{i}: \mathcal{M}_{i} \stackrel{w}{\longrightarrow} \mathcal{M}$ defined by $h_{i}(m)=c_{m}^{\mathcal{M}}$ is a weak elementary embedding.

Remark 4.14. On the basis of the above lemma, call $\left\{\mathcal{M}_{\infty},\left(h_{i}: \mathcal{M}_{i} \stackrel{w}{\hookrightarrow}\right.\right.$ $\left.\left.\mathcal{M}_{\infty}\right)_{i \in \mathbb{N}}\right\}$ a pseudo-direct limit, since the proof does not guarantee the uniqueness of the desired structure. Hence, it may be easily seen that if $\mathcal{M}_{\infty}$ and $\mathcal{N}_{\infty}$ are two pseudo-direct limit of a direct system, then $\mathcal{M}_{\infty} \equiv_{h} \mathcal{N}_{\infty}$ for some $h: \mathbb{I} \rightarrow \mathbb{I}$.

Theorem 4.15. (Robinson consistency theorem) Let $\mathcal{L}, \mathcal{L}_{1}$ and $\mathcal{L}_{2}$ be three ultrametric languages such that $\mathcal{L}=\mathcal{L}_{1} \cap \mathcal{L}_{2}$. Suppose for $i=1,2, T_{i}$ is a satisfiable $\mathcal{L}_{i}$-theory, both including a linear complete $\mathcal{L}$-theory and $T$. Then, $T_{1} \cup T_{2}$ is satisfiable $\mathcal{L}_{1} \cup \mathcal{L}_{2}$-theory.

Proof. Sine $T_{1}$ and $T_{2}$ are satisfiable theories there are an $\mathcal{L}_{1}$-structure $\mathcal{M}_{0} \models T_{1}$ and an $\mathcal{L}_{2}$-structure $\mathcal{N}_{0} \models T_{2}$. Since $T$ is linear complete, by a similar argument used in the amalgamation property, one may show that wediag $\operatorname{L}_{\mathcal{L}}\left(\mathcal{M}_{0}\right) \cup$ wediag $_{\mathcal{L}_{2}}\left(\mathcal{N}_{0}\right)$ is satisfiable. So, by Lemma 4.8, there exists an $\mathcal{L}_{2}$-structure $\mathcal{N}_{1} \models T_{2}$ together with some elementary $\mathcal{L}$-embedding $f_{0}: \mathcal{M}_{0} \hookrightarrow_{\mathcal{L}} \mathcal{N}_{1}$ and weak elementary $\mathcal{L}_{2}$-embedding $g_{0,1}: \mathcal{N}_{0} \stackrel{w}{\longrightarrow}_{\mathcal{L}_{2}} \mathcal{N}_{1}$.

$\mathcal{N}_{1}$ can be naturally viewed as an $\mathcal{L}_{2}\left(M_{0}\right)$-structure where $c_{m}$ is interpreted by $f_{0}(m)$, for any $m \in M_{0}$. So, for any $\mathcal{L}\left(M_{0}\right)$-sentence $\varphi\left(c_{m_{1}}, \ldots, c_{m_{i}}\right)$, we have

$$
\mathcal{M}_{0} \models \varphi\left(c_{m_{1}}, \ldots, c_{m_{i}}\right) \text { iff } \mathcal{N}_{1} \models \varphi\left(c_{m_{1}}, \ldots, c_{m_{i}}\right) \text {. }
$$

Thus, both $\mathcal{M}_{0}$ and $\mathcal{N}_{1}$ are models of the same linear complete $\mathcal{L}\left(M_{0}\right)$-theory, $T h_{\mathcal{L}\left(M_{0}\right)}\left(\mathcal{M}_{0}\right)$. Hence, a similar argument gives an $\mathcal{L}_{1}$-structure $\mathcal{M}_{1} \models T_{1}$, an elementary $\mathcal{L}$-embedding $g_{1}: \mathcal{N}_{1} \hookrightarrow_{\mathcal{L}} \mathcal{M}_{1}$ and a weak elementary $\mathcal{L}_{1}$-embedding $f_{0,1}: \mathcal{M}_{0} \stackrel{w}{\hookrightarrow} \mathcal{L}_{1} \mathcal{M}_{1}$ such that $g_{1} \circ f_{0}=f_{0,1}$.

By continuing this method, we get the following diagram shown in Figure 1, in which $f_{i, i+1}: \mathcal{M}_{i} \stackrel{w}{\hookrightarrow}_{\mathcal{L}_{1}} \mathcal{M}_{i+1}$ and $g_{i, i+1}: \mathcal{N}_{i} \stackrel{w}{\hookrightarrow}_{\mathcal{L}_{2}} \mathcal{N}_{i+1}$ are weak elementary embeddings.

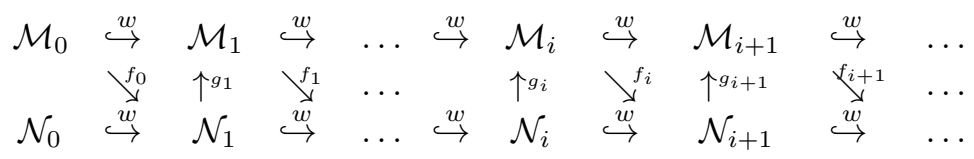

Figure 1. 
Also, $f_{i}: \mathcal{M}_{i} \hookrightarrow_{\mathcal{L}} \mathcal{N}_{i+1}$ and $g_{i}: \mathcal{N}_{i} \hookrightarrow_{\mathcal{L}} \mathcal{M}_{i}$ are elementary embeddings. Furthermore,

$$
\begin{cases}g_{i+1}\left(f_{i}(a)\right)=f_{i, i+1}(a) & \text { for any } a \in M_{i}, \\ f_{i}\left(g_{i}(b)\right)=g_{i, i+1}(b) & \text { for any } b \in N_{i} .\end{cases}
$$

One could construct the above diagram in such a way that for $i \neq j, M_{i} \cap M_{j}=\emptyset$ (resp. $N_{i} \cap N_{j}=\emptyset$ ). For $i \leq j$, define $f_{i, j}: \mathcal{M}_{i} \stackrel{w}{{ }^{w}} \mathcal{L}_{1}\left(\right.$ resp. $g_{i, j}: \mathcal{N}_{i} \stackrel{w}{\hookrightarrow}_{\mathcal{L}_{2}} \mathcal{N}_{j}$ ) so that $\mathcal{F}=\left\{\left(M_{i}\right)_{i \in \mathbb{N}},\left(f_{i, j}\right)_{i \leq j \in \mathbb{N}}\right\}$ (resp. $\mathcal{G}=\left\{\left(N_{i}\right)_{i \in \mathbb{N}},\left(g_{i, j}\right)_{i \leq j \in \mathbb{N}}\right\}$ ) forms a direct system.

Now, take pseudo-direct limits $\left\{\mathcal{M}_{\infty},\left(h_{i}: \mathcal{M}_{i} \stackrel{w}{\hookrightarrow} \mathcal{M}_{\infty}\right)_{i \in \mathbb{N}}\right\}$ and $\left\{\mathcal{N}_{\infty},\left(k_{i}\right.\right.$ : $\left.\left.\mathcal{N}_{i} \stackrel{w}{\rightarrow} \mathcal{N}_{\infty}\right)_{i \in \mathbb{N}}\right\}$ whose underlying sets are $\lim _{\mathcal{F}} M_{i}$ and $\lim _{\mathcal{G}} N_{i}$, respectively.

Define $H: M_{\infty} \rightarrow N_{\infty}$ by $H\left([a]_{\mathcal{F}}\right)=\left[f_{i}(a)\right]_{\mathcal{G}}$, for $a \in M_{i}$. By $(*)$, the function $H$ is well-defined. To see that $H$ is surjective, let $[b]_{\mathcal{G}} \in N$. By the construction of $\mathcal{N}$, there is $j \in \mathbb{N}$ such that $b \in N_{j}$. Set $b^{\prime}=g_{j, j+1}(b)$ and $a=g_{j+1}\left(b^{\prime}\right)$. Then, we have $H\left([a]_{\mathcal{F}}\right)=\left[f_{j+1}(a)\right]_{\mathcal{G}}$. This means

$$
f_{j+1}(a)=f_{j+1}\left(g_{j+1}\left(b^{\prime}\right)\right)=g_{j+1, j+2}\left(b^{\prime}\right)=g_{j+1, j+2}\left(g_{j, j+1}(b)\right)=g_{j, j+2}(b) .
$$

So, $f_{j+1}(a) \equiv_{\mathcal{G}} b$ and therefore, $H\left([a]_{\mathcal{F}}\right)=[b]_{\mathcal{G}}$.

Moreover, for every $\mathcal{L}$-formula $\varphi(\bar{x})$ and $[a]_{\mathcal{F}} \in M_{\infty}, \varphi^{\mathcal{M}_{\infty}}\left([a]_{\mathcal{F}}\right) \approx \varphi^{\mathcal{N}_{\infty}}\left(H\left([a]_{\mathcal{F}}\right)\right)$, since

$$
\varphi^{\mathcal{M}_{\infty}}\left([a]_{\mathcal{F}}\right) \approx \varphi^{\mathcal{M}_{n}}(a)=\varphi^{\mathcal{N}_{n+1}}\left(f_{n}(a)\right) \approx \varphi^{\mathcal{N}_{\infty}}\left(H\left([a]_{\mathcal{F}}\right)\right) .
$$

Therefore, for some $h: \mathbb{I} \rightarrow \mathbb{I}$, we replace $\mathcal{N}_{\infty}$ by another $\mathcal{L}_{2}$-structure $\mathcal{N}$ such that $\mathcal{N} \equiv_{h} \mathcal{N}_{\infty}$ and $\varphi^{\mathcal{N}}\left(H\left([a]_{\mathcal{F}}\right)\right)=h\left(\varphi^{\mathcal{N}_{\infty}}\left(H\left([a]_{\mathcal{F}}\right)\right)=\varphi^{\mathcal{M}_{\infty}}\left([a]_{\mathcal{F}}\right)\right.$. Moreover, for $x, y \in M_{\infty}, d^{\mathcal{M}_{\infty}}(x, y)=d^{\mathcal{N}}(H(x), H(y))$. So, $H$ is one to one.

Therefore, $H$ is an $\mathcal{L}$-isomorphism of $\mathcal{M}_{\infty}$ onto $\mathcal{N}$. Hence, there is an ultrametric $\mathcal{L}_{1} \cup \mathcal{L}_{2}$-structure $\mathcal{P}$ such that $\left.\mathcal{P}\right|_{\mathcal{L}_{1}}=\mathcal{M}_{\infty}$ and $\left.\mathcal{P}\right|_{\mathcal{L}_{2}} \cong \mathcal{N}$. Thus, $\mathcal{P} \models T_{1} \cup T_{2}$.

Remark 4.16. The translation given in Remark 2.25 can be easily applied to the present context to show that the Robinson joint consistency theorem (Theorem 4.15) holds for (fuzzy) first-order rational Gödel logic with equality.

\section{Future Works}

One should further develop the ultrametric logic to make it more accessible for axiomatizing interesting mathematical structures such as valued fields and $p$ adic fields. To this end, one may consider a more general value space and perhaps some additional logical connectives. Any possible approach should allow us to extend the basic results such as completeness and compactness theorems even for uncountable languages. Therefore, the extended semantic should also include "uncountable" structures.

Another interesting topic of research is to study the Robinson joint consistency theorem for first-order (rational) Gödel logic.

\section{Funding}

The second author was partially supported by a grant from IPM, grant number 92030118. 


\section{References}

[1] Matthias Baaz, Norbert Preining, and Richard Zach. First-order Gödel logics. Annals of Pure and Applied Logic, 147(1):23-47, 2007.

[2] Lawrence Peter Belluce and Chen Chung Chang. A weak completeness theorem for infinite valued first-order logic. Journal of Symbolic Logic, 28(1):43-50, 1963.

[3] Itaï Ben Yaacov, Alexander Berenstein, C. Ward Henson, and Alexander Usvyatsov. Model theory for metric structures. in Model theory with applications to algebra and analysis, Volume 2, London Math. Soc. Lecture Note Ser., 350:315-427, 2008.

[4] Itaï Ben Yaacov and Arthur Paul Pedersen. A proof of completeness for continuous first-order logic. Journal of Symbolic Logic, 75(1):168-190, 2010.

[5] Itaï Ben Yaacov and Alexander Usvyatsov. Continuous first order logic and local stability. Transactions of the American Mathematical Society, 362(10):5213-5259, 2010.

[6] Chen Chung Chang. A new proof of the completeness of the Lukasiewicz axioms. Transactions of the American Mathematical Society, 93(1):74-80, 1959.

[7] Chen Chung Chang and H Jerome Keisler. Continuous Model Theory, volume 58. Princeton: Princeton University Press, 1966.

[8] Michael Dummett. A propositional calculus with denumerable matrix. Journal of Symbolic Logic, 24(2):97-106, 1959.

[9] Francesc Esteva, Lluís Godo, and Carles Noguera. First-order t-norm based fuzzy logics with truth-constants: distinguished semantics and completeness properties. Annals of Pure and Applied Logic, 161(2):185-202, 2009.

[10] Petr Hájek. Metamathematics of Fuzzy Logic, volume 4. Kluwer Academic Pub, 1998.

[11] Alfred Horn. Logic with truth values in a linearly ordered Heyting algebra. Journal of Symbolic Logic, 34(3):395-408, 1969.

[12] Jan Łukasiewicz. O logice trójwartościowej (on three-valued logic). Ruch filozoficzny, 5:170-171, 1920.

[13] Jan Pavelka. On fuzzy logic i, ii, iii. Mathematical Logic Quarterly, 25(36,7-12,25-29):45-52,119-134,447-464, 1979.

[14] Alan Rose and J Barkley Rosser. Fragments of many-valued statement calculi. Transactions of the American Mathematical Society, 87(1):1-53, 1958. 